Commun. Math. Phys. 171, $139-158$ (1995)

\title{
The Tangent Bundle of a Calabi-Yau Manifold - Deformations and Restriction to Rational Curves
}

\author{
D. Huybrechts
}

Max-Planck-Institut für Mathematik, Gottfried-Claren-Str. 26, 53225 Bonn, Germany; Institute for Advanced Study, School of Mathematics, Olden Lane, Princeton, 08540 NJ, USA

Received: 10 May 1994 / in revised form: 27 October 1994

\begin{abstract}
The tangent bundle $\mathscr{T}_{X}$ of a Calabi-Yau threefold $X$ is the only known example of a stable bundle with non-trivial restriction to any rational curve on $X$. By deforming the direct sum of $\mathscr{T}_{X}$ and the trivial line bundle one can try to obtain new examples. We use algebro-geometric techniques to derive results in this direction. The relation to the finiteness of rational curves on Calabi-Yau threefolds is discussed.
\end{abstract}

\section{Introduction}

In [W] Witten posed the following question:

Can one deform the vector bundle $\mathscr{T}_{X} \oplus \mathcal{O}_{X}$ to a stable vector bundle whose restriction to any rational curve is nontrivial?

Here $\mathscr{T}_{X}$ is the tangent bundle of a Calabi-Yau threefold $X$ and $\mathcal{O}_{X}$ is the trivial line bundle on it. He showed that such deformations are of significance in string theory (existence of flat directions in the superpotential). In fact, $\left(X, \mathscr{T}_{X}\right)$ seems to be the only known example for a pair $(X, E)$ consisting of a Calabi-Yau manifold $X$ and a stable vector bundle $E$ with nontrivial restriction to any rational curve. A positive answer to the above question would provide an example with a rank four bundle whose Chern classes are those of $X$. This problem and certain generalizations of it were also formulated in problem 77 in Yau's recent problem list [Y].

This paper grew out of the attempt to understand the problem in algebrogeometric terms and to use the available techniques in deformation theory to derive some first results in special cases. In particular we prove:

- Let $X$ be embedded as a hypersurface and assume that it can be deformed in the ambient space to another Calabi-Yau threefold $X^{\prime}$ not isomorphic to $X$ with $X \cap X^{\prime} \neq \emptyset$ (e.g. $X$ is a complete intersection). Then $\mathscr{T}_{X} \oplus \mathcal{O}_{X}$ can be deformed to a stable bundle (1.3).

- For the generic quintic $X \subset \mathbf{P}_{4}$ there exists a stable deformation of $\mathscr{T}_{X} \oplus$ $\mathcal{O}_{X}$ whose restriction to all lines, i.e. rational curves of degree one, is not trivial (3.3). 
For a fixed $(-1,-1)$-curve on a quintic it is easy to find a stable deformation of $\mathscr{T}_{X} \oplus \mathcal{O}_{X}$ with non-trivial restriction to this curve. Since Clemens' conjecture [C] is still open, we can only prove

- For any quintic and a fixed rational curve $\mathbf{P}_{1} \longrightarrow X \subset \mathbf{P}_{4}$ of degree $<20$ the bundle $\mathscr{T}_{X} \oplus \mathcal{O}_{X}$ admits a stable deformation with non-trivial restriction to this curve (3.3).

The infinitesimal deformations of $\mathscr{T}_{X} \oplus \mathcal{O}_{X}$ are parametrized by the vector space

$$
H^{1}\left(X, \mathscr{E} n d\left(\mathscr{T}_{X} \oplus \mathcal{O}_{X}\right)\right) \cong H^{1}\left(X, \mathscr{E} n d\left(\mathscr{T}_{X}\right)\right) \oplus H^{1}\left(X, \Omega_{X}\right) \oplus H^{1}\left(X, \mathscr{T}_{X}\right)
$$

The spaces $H^{1}\left(X, \Omega_{X}\right)$ and $H^{1}\left(X, \mathscr{T}_{X}\right)$ have been intensively studied in the framework of mirror symmetry, whereas, in general, nothing seems to be known about the third space $H^{1}\left(X, \mathscr{E} n d\left(\mathscr{T}_{X}\right)\right)$ on the right-hand side.

We try to attack the problem by introducing a vector bundle $F$. Choosing an ample line bundle $\mathscr{L}$ on $X$, or a Kähler metric $\omega$, we can define a vector bundle $F$ by means of an extension

$$
0 \longrightarrow \mathcal{O}_{X} \longrightarrow F \longrightarrow \mathscr{T}_{X} \longrightarrow 0
$$

given by $c_{1}(\mathscr{L}) \in H^{1}\left(X, \Omega_{X}\right)$ (resp. $[\omega] \in H^{1}\left(X, \Omega_{X}\right)$ ). If $F$ is associated to $\mathscr{L}$ it can be interpreted as $F=\mathscr{D}^{1}(\mathscr{L})$, the sheaf of differential operators of order $\leq 1$ on $\mathscr{L}$. The above sequence in this situation is the symbol sequence. The vector bundle $F$ is the natural bundle associated to the Kähler manifold $(X, \omega)$. It can also be regarded as a deformation of $\mathscr{T}_{X} \oplus \mathcal{O}_{X}$ and, in fact, any neighbourhood of $\mathscr{T}_{X} \oplus \mathcal{O}_{X}$ in its deformation space contains $F$. In particular, small deformations of $F$ also represent deformations of $\mathscr{T}_{X} \oplus \mathcal{O}_{X}$ itself.

An analogous problem was treated in [Ti2] in the case of a Fano $n$-fold which is Kähler-Einstein. Tian showed that the extension

$$
0 \longrightarrow \mathcal{O}_{X} \longrightarrow F \longrightarrow \mathscr{T}_{X} \longrightarrow 0
$$

given by $c_{1}(X)$ admits a Hermite-Yang-Mills metric. Such a metric cannot exist in the case of a Calabi-Yau manifold but after deforming the holomorphic structure of $F$. This is essentially the above question.

In Sect. 1 we consider the special case of a Calabi-Yau threefold deforming as a hypersurface to a non-isomorphic Calabi-Yau manifold. Here $F$ is described in terms of the sheaf of logarithmic differential forms on the ambient space. We will explain how a stable deformation of $F$ can be constructed by means of a deformation of $X$. Sect. 2 is devoted to the study of the deformations of $\mathscr{T}_{X}$ and $\mathscr{T}_{X} \oplus \mathcal{O}_{X}$ in the general case. Motivated by Sect. 1 we define a map $\Xi: \operatorname{De} f(F) \longrightarrow \operatorname{De} f(X)$ between the space of deformations of $F$ on $X$ and of $X$ itself whose tangent map $\xi: H^{1}(X, \mathscr{E} n d(F)) \longrightarrow H^{1}\left(X, \mathscr{T}_{X}\right)$, under an additional assumption (9), is shown to be surjective. Easy arguments in deformation theory (cf. Appendix B) show that $\mathscr{T}_{X} \oplus \mathcal{O}_{X}$ admits a stable deformation if the image of $\Xi$ is of positive dimension. The surjectivity of $\xi$ says in particular that $\mathscr{T}_{X} \oplus \mathcal{O}_{X}$ infinitesimally deforms to a stable bundle. The results will be applied to complete intersections, where $\Xi$ itself is surjective, and compared with Sect. 1 in the case of a quintic hypersurface.

The problem of how to control the restriction of deformations of $\mathscr{T}_{X} \oplus \mathcal{O}_{X}$ to rational curves contained in the Calabi-Yau manifold is dealt with in Sect. 3. It will be explained that this problem is closely related to Clemens' conjecture about 
the finiteness of rational curves on the generic quintic. A reformulation of this conjecture says that for the generic quintic $X$ and any rational curve $C \subset X$ the cohomology $H^{1}\left(C,\left.\mathscr{T}_{X}\right|_{C}\right)$ vanishes. Section 3.1 states that for the generic CalabiYau manifold at least the homomorphism $H^{1}\left(X, \mathscr{T}_{X}\right) \rightarrow H^{1}\left(C,\left.\mathscr{T}_{X}\right|_{C}\right)$ vanishes. The appendices collect some facts about the deformation theory of manifolds and bundles which are used in Sects. 1-3. Some of the results there might be of independent interest (A.3, B.1, B.7). For readers not familiar with deformation theory Appendix A may also serve as an introduction.

Notation: A Calabi-Yau threefold in this paper will be a (mostly projective) three dimensional manifold $X$ with trivial canonical bundle and vanishing $H^{1}\left(X, \mathcal{O}_{X}\right)$. Frequently, we will use the vanishing of $H^{i}\left(X, \mathscr{L}^{n}\right)(i=1,2)$ for any ample line bundle $\mathscr{L}$. This follows from Kodaira's vanishing theorem.

\section{Logarithmic Differentials}

We start out with the following

Proposition 1.1. Let $\mathscr{L}$ be a line bundle on a smooth manifold $Z$ and $f, f^{\prime}$ be sections of $\mathscr{L}$ defining two distinct smooth irreducible hypersurfaces $X^{\prime}, X \subset Z$. Then there exist two families of vector bundles $\left\{G_{t}\right\}$ and $\left\{G_{t}^{\prime}\right\}$ on $X$ with the following properties:

i) $G_{t} \cong G_{t}^{\prime}$ for $t \neq 0$.

ii) $G_{0}$ and $G_{0}^{\prime}$ are the extensions of $\Omega_{X}$ by $\mathcal{O}_{X}$ given by $\delta\left(\left.f^{\prime}\right|_{X}\right) \in H^{1}\left(X, \mathscr{T}_{X}\right)$ and of $\mathcal{O}_{X}$ by $\Omega_{X}$ given by $c_{1}\left(\mathcal{O}_{X}(X)\right) \in H^{1}\left(X, \Omega_{X}\right)$, resp.

Here $\delta$ denotes the boundary homomorphism $H^{0}(X, \mathcal{O}(X)) \rightarrow H^{1}\left(X, \mathscr{T}_{X}\right)$ given by the normal bundle sequence $\left.0 \longrightarrow \mathscr{T}_{X} \longrightarrow \mathscr{T}_{Z}\right|_{X} \longrightarrow \mathcal{O}_{X}(X) \longrightarrow 0$ and the isomorphism $\left.\mathscr{L}\right|_{X} \cong \mathcal{O}_{X}(X)$. In order to prove the proposition, we first have to introduce some notation and basic diagrams. Let $Z$ be an arbitrary smooth manifold and $X^{\prime} \subset Z$ a smooth irreducible hypersurface. The sheaf $\Omega_{Z}\left\langle X^{\prime}\right\rangle$ of differentials with logarithmic poles along $X^{\prime}$ is locally generated by $d x_{1} / x_{1}, d x_{2}, \ldots, d x_{n}$, where $\left(x_{1}, \ldots, x_{n}\right)$ is a local chart and $x_{1}=0$ is the equation for $X^{\prime}$. The sheaf $\Omega_{Z}\left\langle X^{\prime}\right\rangle$ is in fact locally free and fits into the following two exact sequences.

$$
\begin{gathered}
0 \longrightarrow \Omega_{Z} \longrightarrow \Omega_{Z}\left\langle X^{\prime}\right\rangle \longrightarrow \mathcal{O}_{X^{\prime}} \longrightarrow 0, \\
0 \longrightarrow \Omega_{Z}\left\langle X^{\prime}\right\rangle \longrightarrow \Omega_{Z}\left(X^{\prime}\right) \longrightarrow \Omega_{X^{\prime}}\left(X^{\prime}\right) \longrightarrow 0 .
\end{gathered}
$$

The homomorphism $\Omega_{Z}\left\langle X^{\prime}\right\rangle \rightarrow \mathcal{O}_{X^{\prime}}$ is locally given by $\left.\sum f_{i} d x_{i} \mapsto\left(x_{1} \cdot f_{1}\right)\right|_{X^{\prime}}$. The surjection in (2) is by definition the canonical homomorphism $\left.\Omega_{Z} \rightarrow \Omega_{Z}\right|_{X^{\prime}} \rightarrow \Omega_{X^{\prime}}$ twisted by $\mathcal{O}_{Z}\left(X^{\prime}\right)$.

Let $X$ be another smooth irreducible hypersurface in $Z$. How do the sequences (1) and (2) restrict to $X$ ? We treat the cases $X=X^{\prime}$ and $X \neq X^{\prime}$ separately.

First, let $X=X^{\prime}$. Then (1) $\otimes \mathcal{O}_{X}$ splits into the two short exact sequences

$$
\left.0 \longrightarrow \mathcal{O}_{X}(-X) \longrightarrow \Omega_{Z}\right|_{X} \longrightarrow \Omega_{X} \longrightarrow 0
$$


and

$$
\left.0 \longrightarrow \Omega_{X} \longrightarrow \Omega_{Z}\langle X\rangle\right|_{X} \longrightarrow \mathcal{O}_{X} \longrightarrow 0 \text {. }
$$

Use $\mathscr{T} \operatorname{or}_{Z}\left(\mathcal{O}_{X}, \mathcal{O}_{X}\right) \cong \mathscr{O}_{X}(-X)$ and the local description of the homomorphism $\Omega_{Z} \rightarrow \Omega_{Z}\left\langle X^{\prime}\right\rangle$. Analogously, (2) $\otimes \mathcal{O}_{X}$ splits into

$$
\left.0 \longrightarrow \Omega_{X} \longrightarrow \Omega_{Z}\langle X\rangle\right|_{X} \longrightarrow \mathcal{O}_{X} \longrightarrow 0
$$

and

$$
\left.0 \longrightarrow \mathcal{O}_{X} \longrightarrow \Omega_{Z}(X)\right|_{X} \longrightarrow \Omega_{X}(X) \longrightarrow 0 \text {. }
$$

Obviously, (3) and (6) $\otimes \mathcal{O}_{X}(-X)$ are just the dual of the normal bundle sequence of $X \subset Z$. It is also easy to verify, that (4) and (5) as extensions of $\mathcal{O}_{X}$ by $\Omega_{X}$ are given by $c_{1}\left(\mathcal{O}_{X}(X)\right) \in H^{1}\left(X, \Omega_{X}\right)$ (up to multiplication by non-zero scalars).

Now, let $X \neq X^{\prime}$. Then the restrictions of (1) and (2) to $X$ remain exact (use $\left.\mathscr{T}^{o r_{Z}}\left(\mathcal{O}_{X^{\prime}}, \mathcal{O}_{X}\right)=0\right)$. They fit into the commutative diagram:

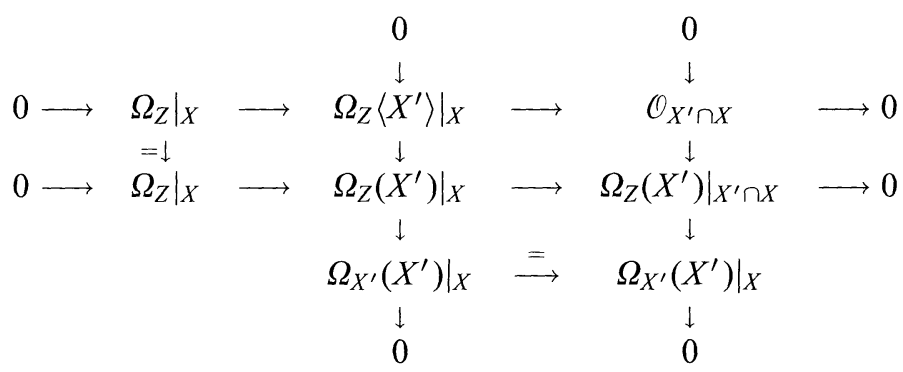

Equivalently, we have

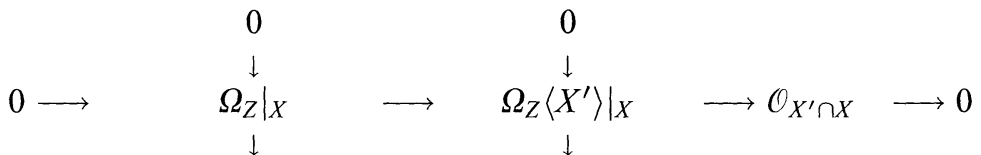

$$
\begin{aligned}
& \left.\left.\Omega_{Z}\left(X^{\prime}\right)\right|_{X} \stackrel{=}{\longrightarrow} \Omega_{Z}\left(X^{\prime}\right)\right|_{X} \\
& 0 \longrightarrow \mathcal{O}_{X^{\prime} \cap X} \longrightarrow \Omega_{Z}\left(\left.\left.\begin{array}{c}
\downarrow \\
X^{\prime}
\end{array}\right|_{X^{\prime} \cap X} \longrightarrow \Omega_{X^{\prime}}\left(\begin{array}{l}
\downarrow \\
X^{\prime}
\end{array}\right)\right|_{X^{\prime} \cap X} \longrightarrow 0\right. \\
& \begin{array}{ll}
\downarrow & \downarrow \\
0 & 0
\end{array}
\end{aligned}
$$

In particular, $\left.\Omega_{Z}\left\langle X^{\prime}\right\rangle\right|_{X}$ is isomorphic to the kernel of the composition $\left.\Omega_{Z}\left(X^{\prime}\right)\right|_{X} \rightarrow$ $\left.\left.\Omega_{Z}\left(X^{\prime}\right)\right|_{X^{\prime} \cap X} \rightarrow \Omega_{X^{\prime}}\left(X^{\prime}\right)\right|_{X^{\prime} \cap X}$. As $X^{\prime}$ approaches $X$ the second homomorphism degenerates to the restriction of the canonical homomorphism $\Omega_{Z} \rightarrow \Omega_{X}$ twisted by $\mathcal{O}\left(X^{\prime}\right)$ to $X^{\prime} \cap X$. For this to happen, $\mathcal{O}(X)$ and $\mathcal{O}\left(X^{\prime}\right)$ must be isomorphic. This gives rise to the following definition.

Definition 1.2. The sheaf $G\left(X^{\prime}, X\right)$ on $X$ is defined as the kernel of the composition

$$
\left.\left.\left.\Omega_{Z}\left(X^{\prime}\right)\right|_{X} \longrightarrow \Omega_{Z}\left(X^{\prime}\right)\right|_{X^{\prime} \cap X} \longrightarrow \Omega_{X}\left(X^{\prime}\right)\right|_{X^{\prime} \cap X}
$$



Using

$G\left(X^{\prime}, X\right)$ replaces $\left.\Omega_{Z}\left\langle X^{\prime}\right\rangle\right|_{X}$ in the diagram above at the limit point $X^{\prime}=X$.

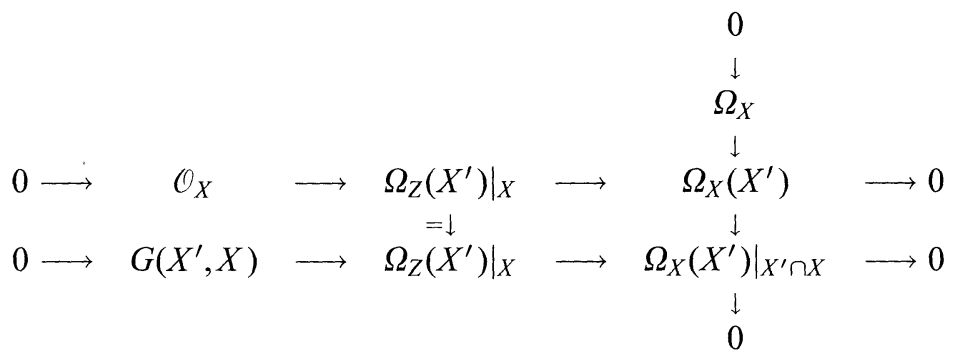

we can write $G\left(X^{\prime}, X\right)$ as an extension

$$
0 \longrightarrow \mathcal{O}_{X} \longrightarrow G\left(X^{\prime}, X\right) \longrightarrow \Omega_{X} \longrightarrow 0
$$

In particular, $G\left(X^{\prime}, X\right)$ is locally free. Now we can prove 1.1 .

Proof of 1.1. $G_{t}^{\prime}$ for all $t$ is defined as $\left.\Omega_{Z}\left\langle X_{t}\right\rangle\right|_{X}$, where $X_{t}$ is the hypersurface given by $t f^{\prime}+(1-t) f$. We define $G_{t}$ as the kernel of the surjection

$$
\left.\left.\left.\Omega_{Z}\right|_{X} \longrightarrow \Omega_{X_{t}}\right|_{X} \longrightarrow \Omega_{X_{t}}\right|_{X^{\prime} \cap X}
$$

twisted by $\mathcal{O}\left(X^{\prime}\right)$. In particular, $\left.G_{0}^{\prime} \cong \Omega_{Z}\langle X\rangle\right|_{X}, G_{0} \cong G\left(X^{\prime}, X\right)$ and $G_{t} \cong G_{t}^{\prime}$ for $t \neq 0$. It remains to prove that $(7)$ is the extension given by $\delta\left(\left.f^{\prime}\right|_{X}\right)$. First, we dualize the diagram above and obtain

$$
\begin{aligned}
& \left.0 \longrightarrow \mathscr{T}_{X}\left(-X^{\prime}\right) \longrightarrow \mathscr{T}_{Z}\left(-X^{\prime}\right)\right|_{X} \longrightarrow \mathcal{O}_{X} \longrightarrow 0 \\
& 0 \longrightarrow \quad \begin{array}{l}
\downarrow \\
\mathscr{T}_{X}
\end{array} \longrightarrow G\left(X^{\prime}, X\right)^{*} \longrightarrow \begin{array}{c}
\mathscr{O}_{X} \\
\end{array} \longrightarrow 0 .
\end{aligned}
$$

Thus the extension class $\eta$ of the second sequence is given by the image of $1 \in H^{0}\left(X, \mathcal{O}_{X}\right)$ under $H^{0}\left(X, \mathcal{O}_{X}\right) \stackrel{\delta\left(-X^{\prime}\right)}{\longrightarrow} H^{1}\left(X, \mathscr{T}_{X}\left(-X^{\prime}\right)\right) \stackrel{\cdot f^{\prime}}{\longrightarrow} H^{1}\left(X, \mathscr{T}_{X}\right)$. The commutativity of the diagram

$$
\begin{array}{ccc}
H^{0}\left(X, \mathcal{O}_{X}\right) & \stackrel{\delta\left(-X^{\prime}\right)}{\longrightarrow} & H^{1}\left(X, \mathscr{T}_{X}(-X)\right) \\
\cdot f^{\prime} \downarrow & & \cdot f^{\prime} \downarrow \\
H^{0}\left(X, \mathcal{O}_{X}\left(X^{\prime}\right)\right) & \stackrel{\delta}{\longrightarrow} & H^{1}\left(X, \mathscr{T}_{X}\right)
\end{array}
$$

implies $\delta\left(\left.f^{\prime}\right|_{X}\right)=\eta$.

We are going to apply 1.1 to the case of a Calabi-Yau threefold. Let $X$ be a three-dimensional Calabi-Yau manifold. As explained in the introduction, we try to obtain stable deformation of $\mathscr{T}_{X} \oplus \mathcal{O}_{X}$ by deforming a non-trivial extension

$$
0 \longrightarrow \mathcal{O}_{X} \longrightarrow F \longrightarrow \mathscr{T}_{X} \longrightarrow 0
$$

given by a Kähler class $\omega \in H^{1}\left(X, \Omega_{X}\right)$. As $\mathscr{T}_{X}$ and $\mathcal{O}_{X}$ are stable vector bundles of the same slope the bundle $F$ is semistable. Moreover, in order to deform $F$ to a stable bundle it is sufficient to find a small deformation $F^{\prime}$ of $F$ with $H^{0}\left(X, F^{\prime}\right) \neq 0$. Here we use the fact that $\mathcal{O}$ has no deformations (for this kind of arguments $\mathrm{cp}$. Appendix B). 
Corollary 1.3. Let $X$ be a Calabi-Yau manifold embedded as a hypersurface in a manifold $Z$ such that

- $0 \neq c_{1}\left(\left.\mathcal{O}(X)\right|_{X}\right) \in H^{1}\left(X, \Omega_{X}\right)$

- $\delta: H^{0}\left(X,\left.\mathcal{O}(X)\right|_{X}\right) \rightarrow H^{1}\left(X, \mathscr{T}_{X}\right)$ does not vanish.

Then $\mathscr{T}_{X} \oplus \mathcal{O}_{X}$ deforms to a stable bundle.

Proof. Choose $f^{\prime} \in H^{0}\left(Z, \mathcal{O}_{Z}(X)\right)$ such that $\delta\left(f^{\prime} \mid X\right) \neq 0$ and apply 1.1. Consider $G_{t}^{\prime *}$ as a deformation of $F$. Since $H^{0}\left(X, G_{0}^{*}\right)=0$, we have $H^{0}\left(X, G_{t}^{* *}\right)=0$ for small $t \neq 0$. Hence, for small $t \neq 0$ the bundle $G_{t}^{*}$ is a stable deformation of $F$.

Remark. o) In most of the cases $Z$ will be a Fano fourfold with $K_{Z}^{*} \cong \mathcal{O}_{Z}(X)$.

i) Examples of Calabi-Yau threefolds satisfying the assumptions of the corollary are all complete intersections in products of projective spaces.

ii) From the proof we can also see that the rank four bundle $\Omega_{Z}\left\langle X_{t}\right\rangle$ on $Z$ is stable with respect to $\mathcal{O}(X)$. This has the flavour of [Ti2] Theorem 5.1.

Note that the two non-trivial extensions

$$
0 \longrightarrow \Omega_{X} \longrightarrow F^{*} \longrightarrow \mathcal{O}_{X} \longrightarrow 0
$$

and

$$
0 \longrightarrow \mathcal{O}_{X} \longrightarrow G\left(X^{\prime}, X\right) \longrightarrow \Omega_{X} \longrightarrow 0
$$

both define simple vector bundles. Under the assumptions of the corollary they define non-separated points in the moduli space of simple bundles. In this vein, $\mathscr{T}_{X} \oplus \mathcal{O}_{X}$ deforms to a stable bundle if two extensions of the above form define nonseparated points in the moduli space of simple bundles. By a result of Norton [No] for non-separated points there exist non-trivial homomorphisms $\varphi: G\left(X^{\prime}, X\right) \rightarrow F^{*}$ and $\xi: F^{*} \rightarrow G\left(X^{\prime}, X\right)$ with $\varphi \circ \xi=0$ and $\xi \circ \varphi=0$. These, obviously, always exist for extensions of the form above, e.g. take the composition $G\left(X^{\prime}, X\right) \rightarrow \Omega_{X} \rightarrow F^{*}$ and $F^{*} \rightarrow \mathcal{O}_{X} \rightarrow G\left(X^{\prime}, X\right)$. But in general the existence of such homomorphisms is not enough to conclude that they define non-separated points in the moduli space.

We want to emphasize once more that the stable deformations of $\mathscr{T}_{X} \oplus \mathcal{O}_{X}$ we obtained are given as $\left.\Omega_{Z}\left\langle X^{\prime}\right\rangle^{*}\right|_{X}$, where $X^{\prime}$ is a non-isomorphic deformation of $X$. In Sect. 2 we will elaborate this idea and explain how deformations of $X$ itself induce, at least infinitesimally and under an additional assumption, stable deformations of $\mathscr{T}_{X} \oplus \mathcal{O}_{X}$. It seems that under this additional assumption the unobstructedness of a Calabi-Yau manifold may help to prove the existence of stable deformations of $\mathscr{T}_{X} \oplus \mathcal{O}_{X}$.

\section{Deformation of $\mathscr{T}_{X}$ and $\mathscr{T}_{X} \oplus \mathcal{O}_{X}$ of a Calabi-Yau Threefold}

Since a Calabi-Yau manifold has unobstructed deformations it is only natural to ask if vector bundles living on the manifold have special deformational properties, too. The most natural bundle on any manifold is the tangent bundle. For a Calabi-Yau manifold it is a stable bundle of degree zero [Ts]. [Mi, Ts]. The tangent bundle of a K3-surface, which could be considered as a two-dimensional Calabi-Yau manifold, has unobstructed deformations. This is due to the vanishing of $H^{2}\left(X, \mathscr{E} n d_{0}\left(\mathscr{T}_{X}\right)\right) \cong$ $H^{0}\left(X, \mathscr{E} n d_{0}\left(\mathscr{T}_{X}\right)\right)^{*}$. 
Almost nothing seems to be known about the deformational behaviour of $\mathscr{T}_{X}$ for a Calabi-Yau threefold. In [EH] and [DGKM] the dimension of $H^{1}\left(X, \mathscr{E} n d\left(\mathscr{T}_{X}\right)\right)$ was computed for a quintic hypersurface, it is 224 in this case, for a bi-cubic hypersurface in $\mathbf{P}_{2} \times \mathbf{P}_{2}$, here it is 176 , and for some other complete intersections in the product of two projective spaces. The following questions seem to be interesting in this context.

Questions. $i)$ Is there a Calabi-Yau threefold with $H^{1}\left(X, \mathscr{E} n d\left(\mathscr{T}_{X}\right)\right)=0$ ?

ii) Is $\operatorname{dim} H^{1}\left(X, \mathscr{E} n d\left(\mathscr{T}_{X}\right)\right)$ invariant under deformation of $X$ ? Even for surfaces I do not know the answer.

iii) Is there a Calabi-Yau threefold such that $\operatorname{De} f\left(\mathscr{T}_{X}\right)$ is not smooth?

For certain Calabi-Yau threefolds the deformations of $\mathscr{T}_{X}$ and $\mathscr{T}_{X} \oplus \mathcal{O}_{X}$ can be constructed in terms of a projective embedding of $X$. The additional assumptions we need are formulated in the following two conditions.

The homomorphism $H^{1}\left(X, \mathscr{T}_{X}\right) \stackrel{\cup c_{1}(\mathscr{L})}{\longrightarrow} H^{2}\left(X, \mathscr{T}_{X} \otimes \Omega_{X}\right)$ vanishes.

The homomorphism $H^{1}\left(X, \mathscr{D}^{1}(\mathscr{L})\right) \stackrel{\cup c_{1}(\mathscr{L})}{\longrightarrow} H^{2}\left(X, \mathscr{D}^{1}(\mathscr{L}) \otimes \Omega_{X}\right)$ vanishes.

For the definition of $\mathscr{D}^{1}(\mathscr{L})$, which is isomorphic to $\mathscr{D}_{0}^{1}(\mathscr{L})$ if $\mathscr{L}$ is of rank one, we refer the reader to Appendix A. We will indicate whenever we use either of these conditions.

Remark. Since for Calabi-Yau manifolds the homomorphism $H^{1}\left(X, \mathscr{D}^{1}(\mathscr{L})\right) \longrightarrow$ $H^{1}\left(X, \mathscr{T}_{X}\right)$ is surjective, (9) implies (8). For complete intersections it is easy to verify (9). In fact, the map in (9) factorizes through $H^{2}\left(X, \mathscr{D}^{1}(\mathscr{L}) \otimes \Omega_{\mathbf{P}_{N}} \mid X\right)$, since $c_{1}(\mathscr{L})=c_{1}\left(\left.\mathcal{O}(1)\right|_{X}\right) \in H^{1}\left(X,\left.\Omega_{\mathbf{P}_{N}}\right|_{X}\right)$. We claim that this space vanishes. Using the diagram

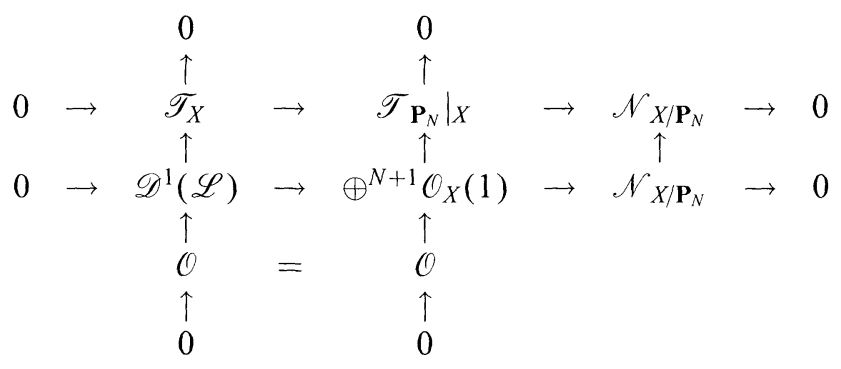

we get the exact sequence

$$
H^{1}\left(X,\left.\Omega_{\mathbf{P}_{N}}\right|_{X} \otimes \mathscr{N}_{X / \mathbf{P}_{N}}\right) \longrightarrow H^{2}\left(X,\left.\mathscr{D}^{1}(\mathscr{L}) \otimes \Omega_{\mathbf{P}_{N}}\right|_{X}\right) \longrightarrow H^{2}\left(X,\left.\oplus^{N+1} \Omega_{\mathbf{P}_{N}}(1)\right|_{X}\right)
$$

$H^{2}\left(X,\left.\Omega_{\mathbf{P}_{N}}(1)\right|_{X}\right)$ is zero by Kodaira's vanishing theorem. $H^{1}\left(X,\left.\Omega_{\mathbf{P}_{N}}\right|_{X} \otimes \mathscr{N}_{X / \mathbf{P}_{N}}\right)$ is zero, since $H^{0}\left(X, \bigoplus^{N+1} \mathscr{N}_{X / \mathbf{P}_{N}}(-1)\right) \longrightarrow H^{0}\left(X, \mathscr{N}_{X / \mathbf{P}_{N}}\right)$ and $H^{1}\left(X, \oplus \mathscr{N}_{X / \mathbf{P}_{N}}\right.$ $(-1))=0$.

Other examples satisfying (9) are provided by double coverings of $\mathbf{P}_{3}$ ramified along a smooth octic. 
The deformations of $\mathscr{T}_{X}$ will be investigated first. Choosing a high power $\mathscr{L}^{n}$ of the ample line bundle $\mathscr{L}$ we obtain a projective embedding $X \subset \mathbf{P}_{N}$ with $\left.\mathscr{L}^{n} \cong \mathcal{O}(1)\right|_{X}$. We want to use the normal bundle sequence

$$
\left.0 \longrightarrow \mathscr{T}_{X} \longrightarrow \mathscr{T}_{\mathbf{P}_{N}}\right|_{X} \longrightarrow \mathscr{N}_{X / \mathbf{P}_{N}} \longrightarrow 0
$$

to construct deformations of $\mathscr{T}_{X}$ by deforming the quotient $\left.\mathscr{T}_{\mathbf{P}_{N}}\right|_{X} \longrightarrow \mathscr{N}_{X \mid \mathbf{P}_{N}}$. Since $X$ is a Calabi-Yau manifold, $H^{1}\left(X, \mathscr{E} n d\left(\mathscr{T}_{\mathbf{P}_{N}} \mid X\right)\right)=0$. In fact, the next lemma shows that under (8), at least infinitesimally, all deformations of $\mathscr{T}_{X}$ are obtained in this way.

Lemma 2.1. If $H^{2}\left(X, \mathscr{T}_{X} \otimes \mathscr{L}^{-n}\right)=0$ and (8) holds true, then $\operatorname{Hom}\left(\mathscr{T}_{X}, \mathscr{N}_{X \mid \mathbf{P}_{N}}\right)$ $\longrightarrow H^{1}\left(X, \mathscr{E} n d\left(\mathscr{T}_{X}\right)\right)$ is surjective. This is the tangent map of the locally defined morphism of the Quot-scheme parametrizing all quotients of $\left.\mathscr{T}_{\mathbf{P}_{N}}\right|_{X}$ with fixed

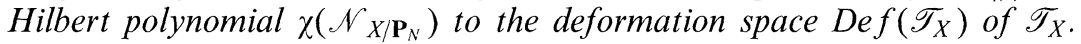

Proof. The surjectivity is equivalent to the vanishing of the homomorphism

$$
H^{1}\left(X, \mathscr{E} n d\left(\mathscr{T}_{X}\right)\right) \longrightarrow H^{1}\left(X, \mathscr{H} \operatorname{om}\left(\mathscr{T}_{X},\left.\mathscr{T}_{\mathbf{P}_{N}}\right|_{X}\right)\right)
$$

By Serre duality it is enough to show that $H^{2}\left(X, \mathscr{H} \operatorname{om}\left(\left.\mathscr{T}_{\mathbf{P}_{N}}\right|_{X}, \mathscr{T}_{X}\right)\right) \longrightarrow$ $H^{2}\left(X, \mathscr{E} n d\left(\mathscr{T}_{X}\right)\right)$ vanishes. The natural homomorphism

$$
H^{1}\left(X, \mathscr{T}_{X}\right) \longrightarrow H^{2}\left(X, \mathscr{H} \operatorname{om}\left(\left.\mathscr{T}_{\mathbf{P}_{N}}\right|_{X}, \mathscr{T}_{X}\right)\right)
$$

which is given by applying $\operatorname{Hom}\left(, \mathscr{T}_{X}\right)$ to the Euler sequence

$$
\left.0 \longrightarrow \mathcal{O}_{X} \longrightarrow \oplus^{N+1} \mathscr{L}^{n} \longrightarrow \mathscr{T}_{\mathbf{P}_{N}}\right|_{X} \longrightarrow 0
$$

is surjective, since the cokernel of this map is contained in $\bigoplus^{N+1} H^{2}\left(X, \mathscr{T}_{X} \otimes\right.$ $\left.\mathscr{L}^{-n}\right)$. Therefore, it is enough to ensure that the composition $H^{1}\left(X, \mathscr{T}_{X}\right) \longrightarrow$ $H^{2}\left(X, \mathscr{E} n d\left(\mathscr{T}_{X}\right)\right)$ is the zero map. This is exactly (8).

Since for a complete intersection $X \subset \mathbf{P}_{N}$ the normal bundle $\mathscr{N}_{X / \mathbf{P}_{N}}$ is isomorphic to $\bigoplus \mathscr{L}^{n_{t}}$, where $\left.\mathscr{L} \cong \mathcal{O}_{\mathbf{P}_{N}}(1)\right|_{X}$, we get

Corollary 2.2. If $X$ is a complete intersection Calabi-Yau threefold, then the tangent bundle $\mathscr{T}_{X}$ has unobstructed deformations.

Proof. We know that $H^{0}\left(\mathbf{P}_{N}, \mathcal{O}_{\mathbf{P}_{N}}(1)\right) \rightarrow H^{0}(X, \mathscr{L})$ is surjective. By Serre duality we see that $H^{3}\left(X, \mathscr{L}^{*}\right) \rightarrow H^{3}\left(X, \bigoplus^{N+1} \mathcal{O}_{X}\right)$ is injective. Hence $H^{2}\left(X,\left.\mathscr{T}_{\mathbf{P}_{N}}\right|_{X} \otimes \mathscr{L}^{*}\right)$ vanishes. So we can apply 2.1. Since $\mathscr{N}_{X / \mathbf{P}_{N}} \cong \bigoplus \mathscr{L}^{n_{1}}$, the normal bundle itself is a rigid bundle on $X$, i.e. $\operatorname{Ext}^{1}\left(\mathscr{N}_{X / \mathbf{P}_{N}}, \mathscr{N}_{X / \mathbf{P}_{N}}\right)=0$. Hence all the deformations of the quotients $\mathscr{T}_{\mathbf{P}_{N}} \mid X \longrightarrow \mathscr{N}_{X / \mathbf{P}_{N}}$ are induced by changing the maps only. In particular, the corresponding Quot-scheme is smooth. Hence by 2.1 the deformation space $\operatorname{De} f\left(\mathscr{T}_{X}\right)$ is smooth.

Remark. This lemma has an interesting global aspect. Moduli spaces of stable vector bundles on a $\mathrm{K} 3$-surface are not unirational. Whereas in our situation the moduli space parametrizing deformations of the tangent bundle is unirational, since it is dominated by the space of all homomorphisms of $\left.\mathscr{T}_{\mathbf{P}_{N}}\right|_{X}$ to $\mathscr{N}_{\mathbf{P}_{N} / X}$. 
We want to come back to Witten's question and ask for deformation of $\mathscr{T}_{X} \oplus$ $\mathcal{O}_{X}$. First, we would like to see if there are stable deformations at all. It might be interesting to look at this in a more general setting and to construct stable deformations of a sum of two stable vector bundles on an arbitrary manifold. This problem is dealt with in Appendix B.

Let $F$ be the semistable vector bundle of rank four and degree zero defined as an extension of $\mathscr{T}_{X}$ by $\mathcal{O}_{X}$ by a Kähler class. It encodes information about the Calabi-Yau manifold.

- $H^{1}(X, F) \cong H^{1}\left(X, \mathscr{T}_{X}\right)$ and $H^{2}(X, F) \cong\left(H^{1}\left(X, \Omega_{X}\right) / \omega \mathbb{C}\right)^{*}$

- A smooth rational curve $C \subset X$ is a $(-1,-1)$-curve if and only if $F_{C} \cong$ $(\mathcal{O}(1) \oplus \mathcal{O}(-1))^{\oplus 2}$. (Use that $\left.\omega\right|_{C}$ does not vanish.)

- The infinitesimal deformation space $H^{1}(X, \mathscr{E} n d(F))$ of the bundle $F$ naturally relates $H^{1}\left(X, \mathscr{T}_{X}\right)$ and $H^{1}\left(X, \Omega_{X}\right)$ by the following diagram of exact sequences.

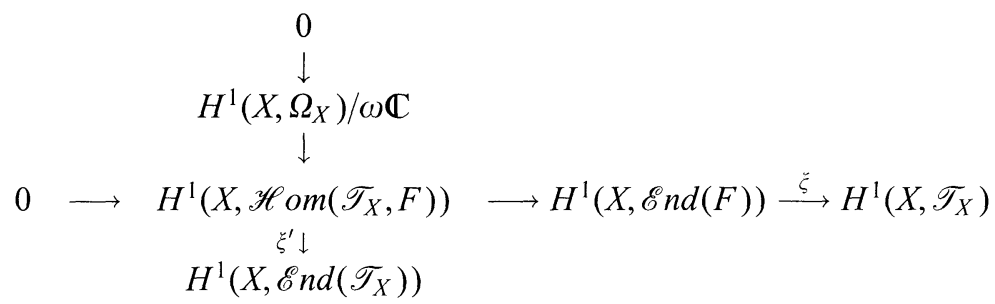

The first two properties are easily derived from the definition. The diagram is produced by applying $\operatorname{Hom}(, F)$ to the short exact sequence defining $F$ and using $H^{1}(X, F) \cong H^{1}\left(X, \mathscr{T}_{X}\right)$. The cokernel of $\xi^{\prime}$ is the image of the homomorphism $H^{1}\left(X, \mathscr{E} n d\left(\mathscr{T}_{X}\right)\right) \rightarrow H^{1}\left(X, \Omega_{X}\right)$ which is dual to $H^{1}\left(X, \mathscr{T}_{X}\right) \stackrel{\cup \omega}{\longrightarrow} H^{2}\left(X, \Omega_{X} \otimes \mathscr{T}_{X}\right)$. If $[\omega]$ is a Hodge class $c_{1}(\mathscr{L})$ such that $(8)$ holds true, then the vertical sequence can be completed to a short exact sequence, i.e. $\xi^{\prime}$ is surjective.

The space $H^{1}(X, \mathscr{E} n d(F))$ parametrizes all infinitesimal deformations of the bundle $F$ on $X$, i.e. it is the tangent space of $\operatorname{De} f(F)$. The cohomology group $H^{1}\left(X, \mathscr{T}_{X}\right)$ parametrizes the infinitesimal deformations of $X$ itself (cf. A). Since a Calabi-Yau manifold has unobstructed deformations and using the Kuranishi description of the deformation spaces (cf. App. A), $\xi: H^{1}(X, \mathscr{E} n d(F)) \longrightarrow H^{1}\left(X, \mathscr{T}_{X}\right)$ induces a map $\Xi: \operatorname{De} f(F) \rightarrow \operatorname{De} f(X)$, between the corresponding deformation spaces, which has $\xi$ as its tangent map. At the first glance this map seems to be quite artificial, but it explains the phenomena of Sect. 1. As a consequence of (B.7) one proves

Corollary 2.3. If the image of $\Xi$ is positive dimensional, then $\mathscr{T}_{X} \oplus \mathcal{O}_{X}$ deforms to a stable bundle.

Using the map $\Xi$ one can reformulate condition (9).

Lemma 2.4. If $[\omega]$ is a Hodge class satisfying (9), then $\Xi$ is a submersion, i.e. $\xi$ is surjective.

We say that $\mathscr{T}_{X} \oplus \mathcal{O}_{X}$ deforms infinitesimally to a stable bundle if $\xi$ does not vanish. 
Remarks. i) Under the assumptions of (1.3) the map $\Xi$ has positive dimensional image. In fact, $\operatorname{dim} \operatorname{Im}(\Xi) \geqq \operatorname{rk}(\delta)$, since $d\left(\Xi\left(\left.\Omega_{Z}\left\langle X_{t}\right\rangle^{*}\right|_{X}\right)\right) / d t=\delta(f)$.

ii) For complete intersection one can do better. On a complete intersection the bundle $F$ has unobstructed deformations. To verify this assertion one first shows that for any Calabi-Yau manifold $\operatorname{Hom}\left(F, \mathscr{N}_{X / \mathbf{P}_{N}}\right) \longrightarrow H^{1}(X, \mathscr{E} n d(F))$ is surjective, i.e. all infinitesimal deformations of $F$ are obtained as the kernel of a map $\oplus^{N+1} \mathscr{L}^{n} \longrightarrow$ $\mathscr{N}_{X / \mathbf{P}_{N}}$. Of course, we assume $n \gg 0$. This is in analogy to $(2.1)$, but here we do not need (8) or (9). For complete intersections the normal bundle $\mathscr{N}_{X / \mathbf{P}_{N}}$ is infinitesimal rigid and the vanishing $H^{1}(X, F(-1))$ holds true (cf. (2.2)).

iii) Moreover, for complete intersections the bundle $\mathscr{T}_{X} \oplus \mathcal{O}_{X}$ has unobstructed deformations, since $h^{1}\left(X, \mathscr{E} n d\left(\mathscr{T}_{X} \oplus \mathcal{O}_{X}\right)\right)=h^{1}(X, \mathscr{E} n d(F))+1$ and the image of the map $\mathbb{C} \backslash\{0\} \times \operatorname{Def}(F) \rightarrow \operatorname{Def}\left(\mathscr{T}_{X} \oplus \mathcal{O}_{X}\right)$, induced by $(t, 0) \mapsto[t \omega]$, is of dimension $1+\operatorname{dim} \operatorname{Def}(F)$. (We use that $\operatorname{Def}\left(\mathscr{T}_{X} \oplus \mathcal{O}_{X}\right)$ is still complete at the point

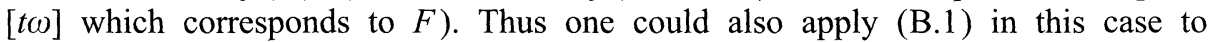
conclude the existence of stable deformations of $\mathscr{T}_{X} \oplus \mathcal{O}_{X}$.

iv) Obviously, (2.2) and ii) go through for Calabi-Yau threefolds $X \subset \mathbf{P}_{N}$ with rigid normal bundle satisfying $(9)$ and $H^{2}\left(X, \mathscr{T}_{X}(-1)\right)=H^{2}(X, F(-1))=0$. Along the same line one can treat complete intersections in the product of projective spaces.

To conclude this section we want to present some further calculations in the case of a quintic hypersurface. In the exact sequence

$$
0 \longrightarrow F \longrightarrow \oplus^{5} \mathscr{L} \longrightarrow \mathscr{L}^{5} \longrightarrow 0
$$

the surjection $\oplus^{5} \mathscr{L} \longrightarrow \mathscr{L}^{5}$ is given by the restriction of the map

$$
\oplus^{5} \mathcal{O}_{\mathbf{P}_{4}}(1) \stackrel{\left(\partial f / \partial x_{0}, \ldots, \partial f / \partial x_{4}\right)}{\longrightarrow} \mathcal{O}_{\mathbf{P}_{4}}(5)
$$

where $X$ is defined by $f \in H^{0}\left(\mathbf{P}_{4}, \mathcal{O}_{\mathbf{P}_{4}}(5)\right)$ and the $x_{i}$ are coordinates of $\mathbf{P}_{4}$. Obviously, this map is also surjective. Hence $F$ is isomorphic to the restriction of a vector bundle $F_{f}$ on $\mathbf{P}_{4}$ which is defined as the kernel of $\left(\partial f / \partial x_{i}\right)$. For any other $f^{\prime} \in H^{0}\left(\mathbf{P}_{4}, \mathcal{O}_{\mathbf{P}_{4}}(5)\right)$ defining a smooth quintic $X^{\prime}$ we also get a bundle $F_{f^{\prime}}$ on $\mathbf{P}_{4}$. Its restriction to $X$ can be regarded as a deformation of $F$. In this way we can locally define a map $\left|\mathcal{O}_{\mathbf{P}_{4}}(5)\right| \longrightarrow \operatorname{De} f(F)$. Note that $F_{\alpha f} \cong F_{f}$ for $\alpha \in \mathbb{C}^{*}$. Less geometrically, it can be described as follows. As we have seen there is a map $\operatorname{Hom}\left(\oplus^{5} \mathscr{L}, \mathscr{L}^{5}\right) \longrightarrow \operatorname{De} f(F)$ which is locally defined near $\left(\partial f / \partial x_{i}\right)$. The natural surjection $\operatorname{Hom}\left(\oplus^{5} \mathcal{O}_{\mathbf{P}_{4}}(1), \mathcal{O}_{\mathbf{P}_{4}}(5)\right) \longrightarrow \operatorname{Hom}\left(\mathcal{O}_{\mathbf{P}_{4}}, \mathcal{O}_{\mathbf{P}_{4}}(5)\right)$ has a section $f^{\prime} \rightarrow\left(\partial f^{\prime} / \partial x_{i}\right)$, which induces the above map $\left|\mathcal{O}_{\mathbf{P}_{4}}(5)\right| \rightarrow \operatorname{De} f(F)$.

Lemma 2.5. Let $\left|\mathcal{O}_{\mathbf{P}_{4}}(5)\right| \longrightarrow \operatorname{De} f(X)$ be the locally defined natural map associating to a quintic its underlying manifold. Then the following diagram commutes:

$$
\begin{aligned}
\left|\mathcal{O}_{\mathbf{P}_{4}}(5)\right| \longrightarrow & \operatorname{Def}(F) \\
& \downarrow \\
& \operatorname{Def}(X) .
\end{aligned}
$$

Proof. This is a consequence of the commutativity of the following two diagrams.

$$
\begin{array}{ccc}
\operatorname{Hom}\left(\oplus^{5} \mathscr{L}, \mathscr{L}^{5}\right) & \longrightarrow & \operatorname{Hom}\left(F, \mathscr{L}^{5}\right) \\
\downarrow & & \downarrow \\
H^{0}\left(X, \mathscr{L}^{5}\right) & \longrightarrow & H^{0}\left(X, \mathscr{L}^{5}\right)
\end{array}
$$


and

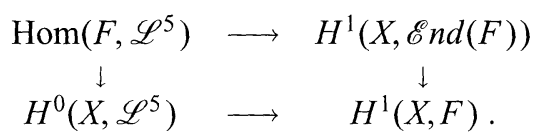

The first one is induced by the injection of $\mathcal{O}_{X}$ into $\oplus^{5} \mathscr{L}$ resp. $F$ and the second one by the boundary operator of

$$
0 \longrightarrow F \longrightarrow \oplus^{5} \mathscr{L} \longrightarrow \mathscr{L}^{5} \longrightarrow 0
$$

Note that $\left|\mathcal{O}_{\mathbf{P}_{4}}(5)\right| \longrightarrow \operatorname{Def}(F)$ does not induce a section of $\Xi$. It is not hard to see that the above map $\left|\mathcal{O}_{\mathbf{P}_{4}}(5)\right| \rightarrow \operatorname{De} f(F)$ coincides with $\left.X^{\prime} \mapsto \Omega_{\mathbf{P}_{4}}\left\langle X^{\prime}\right\rangle^{*}\right|_{X}$, i.e. $\left.\Omega_{\mathbf{P}_{4}}\left\langle X^{\prime}\right\rangle^{*}\right|_{X} \cong F_{f^{\prime}}$ (cf. Sect. 1).

\section{Restriction to Rational Curves}

In the theory of Calabi-Yau manifolds it is important to know if there are rational curves on the manifold, and if, how many. Since $h^{0}\left(X, \mathscr{N}_{C / X}\right)-h^{1}\left(X, \mathscr{N}_{C / X}\right)=0$ by Riemann-Roch, one expects a rational curve $C$ on a Calabi-Yau manifold $X$ to be isolated, i.e. $C$ cannot be deformed in $X$ (cf. Appendix A). Clemens conjectured that the generic quintic in $\mathbf{P}_{4}$ contains only rational curves with normal bundle isomorphic to $\mathcal{O}(-1) \oplus \mathcal{O}(-1)$ (cf. $[\mathrm{C}, \mathrm{K} 1])$. The conjecture has been verified for curves of degree $\leqq 9([\mathrm{~K} 1, \mathrm{KJ}, \mathrm{N}])$. In particular, $(-1,-1)$-curves satisfy $H^{1}\left(C, \mathscr{N}_{C / X}\right)=0$, i.e. they are infinitesimal rigid. Note that $H^{1}\left(C, \mathscr{N}_{C / X}\right)=0$ is equivalent to $H^{1}\left(C,\left.\mathscr{T}_{X}\right|_{C}\right)=0$. We start with the following result.

Theorem 3.1. For the generic Calabi-Yau threefold $X$, i.e. generic in its Kuranishi family, and any rational curve $\varphi: \mathbf{P}_{1} \rightarrow X$, the restriction map $H^{1}\left(X, \mathscr{T}_{X}\right) \rightarrow$ $H^{1}\left(\mathbf{P}_{1}, \varphi^{*}\left(\mathscr{T}_{X}\right)\right)$ vanishes.

Proof. Let $\mathscr{X} \rightarrow T$ be the Kuranishi family of $X$. By [Ka, R, Ti, To] the base space $T$ is smooth (cf. A.1). Let $\operatorname{Hilb}^{d}(\mathscr{X} \rightarrow T)$ be the relative Hilbert scheme (or the Douady space) of morphisms of $\mathbf{P}_{1}$ to the fibres of $\mathscr{X} \rightarrow T$ of degree $d$. Pick an irreducible component $H$ of $\operatorname{Hilb}^{d}(\mathscr{X} \rightarrow T)$. As we are only interested in the generic Calabi-Yau manifold, we can assume that $H$ dominates $T$. Let $U \subset H_{\text {red }}$ be a nonempty smooth open subset, such that $U \rightarrow T$ is smooth. Denote $\mathscr{X} \times{ }_{T} U$ by $\tilde{\mathscr{X}}$. The sequence

$$
0 \longrightarrow \mathscr{T}_{\tilde{X} / U} \longrightarrow \mathscr{T}_{\tilde{X}} \longrightarrow p r_{2}^{*} \tilde{T}_{U} \longrightarrow 0
$$

restricts on $X:=\mathscr{X}_{0}$ to

$$
\left.0 \longrightarrow \mathscr{T}_{X} \longrightarrow \mathscr{T}_{\tilde{X}}\right|_{X} \longrightarrow \mathscr{T}_{U, 0} \otimes \mathcal{O}_{X} \longrightarrow 0
$$

We denote the corresponding extension class by $\eta \in \operatorname{Ext}^{1}\left(\mathscr{T}_{U, 0} \otimes \mathcal{O}_{X}, \mathscr{T}_{X}\right)$. This sequence is in fact the pull-back of the universal extension of $\mathscr{O}_{X}$ by $\mathscr{T}_{X}$ under the Kodaira-Spencer map $\mathscr{T}_{U, 0} \rightarrow H^{1}\left(X, \mathscr{T}_{X}\right)$. Since $U \rightarrow T$ is smooth and $\mathscr{X} \rightarrow T$ is a complete family, the Kodaira-Spencer map is surjective. Therefore, it is enough to prove that (10) splits on every rational curve $\varphi: \mathbf{P}_{1} \rightarrow X$. Let $\tilde{\varphi}: \mathbf{P}_{1} \times U \rightarrow \tilde{X}$ be the universal curve. By $\mathscr{N}_{\varphi}$ and $\mathscr{N}_{\tilde{\varphi}}$ we denote the cokernels of the natural 
homomorphisms $\mathscr{T}_{\mathbf{P}_{1}} \rightarrow \varphi^{*}\left(\mathscr{T}_{X}\right)$ and $\mathscr{T}_{\mathbf{P}_{1}} \rightarrow \tilde{\varphi}^{*}\left(\mathscr{T}_{\tilde{X}}\right)$, resp. If $\varphi$ is an embedding, $\mathscr{N}_{\varphi}$ and $\mathscr{N}_{\tilde{\varphi}}$ are the normal bundles of $\varphi\left(\mathbf{P}_{1}\right)$ in $X$ and of $\varphi\left(\mathbf{P}_{1}\right)$ in $\tilde{\mathscr{X}}$, resp. Thus we obtain the commutative diagram

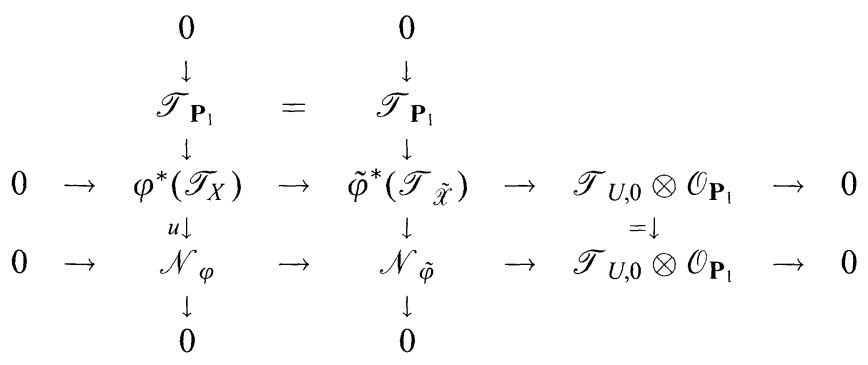

The sequence on the bottom, as an extension of $\mathscr{T}_{U, 0} \otimes \mathcal{O}_{\mathbf{P}_{1}}$ by $\mathscr{N}_{\varphi}$, is given by the image of $u_{*}\left(\varphi^{*}(\eta)\right)$, where $u_{*}: \operatorname{Ext}^{1}\left(\mathscr{T}_{U, 0} \otimes \mathcal{O}_{\mathbf{P}_{1}}, \varphi^{*}\left(\mathscr{T}_{X}\right)\right) \rightarrow \operatorname{Ext}^{1}\left(\mathscr{T}_{U, 0} \otimes \mathcal{O}_{\mathbf{P}_{1}}, \mathscr{N}_{\varphi}\right)$ is induced by $u$. Since $H^{1}\left(\mathbf{P}_{1}, \mathscr{T}_{\mathbf{P}_{1}}\right)=0$, the map $u_{*}$ is injective. Thus it is enough to show that $u_{*}\left(\varphi^{*}((\eta))=0\right.$, i.e. that the bottom sequence of the diagram splits. The homomorphism $\left.\mathscr{T}_{\mathbf{P}_{1} \times U}\right|_{\mathbf{P}_{1} \times\{0\}} \rightarrow \tilde{\varphi}^{*}\left(\mathscr{T}_{\tilde{X}}\right)$ induces a natural map $\mathscr{N}_{\mathbf{P}_{1} \times\{0\} / \mathbf{P}_{1} \times U} \rightarrow$ $\mathscr{N}_{\tilde{\varphi}}$. Since $\mathscr{N}_{\mathbf{P}_{1} \times\{0\} / \mathbf{P}_{1} \times U} \cong \mathscr{T}_{U, 0} \otimes \mathcal{O}_{\mathbf{P}_{1}}$, we can take this map to define the desired splitting.

Remark. Of course, if Clemens' conjecture holds true for $X$ the assertion of the theorem is obvious. On the other hand, one can try to attack the conjecture using this result by proving that the above restriction homomorphism is always surjective. For a quintic this is true if and only if for a curve $\varphi: \mathbf{P}_{1} \rightarrow X \subset \mathbf{P}_{4}$, where $X$ is defined by $f$, the space $H^{0}\left(\mathbf{P}_{1}, \varphi^{*} \mathcal{O}(5)\right)$ is spanned by $\varphi^{*}\left(H^{0}\left(\mathbf{P}_{4}, \mathcal{O}(5)\right)\right)$ and $\sum_{i=0}^{4} H^{0}\left(\mathbf{P}_{1}, \varphi^{*} \mathcal{O}(1)\right) \varphi^{*}\left(\frac{\partial f}{\partial x_{1}}\right)$. In the cases of a quintic, of a complete intersection of type $(3,3)$ and of a complete intersection of type $(2,4),(2,2,3)$ or $(2,2,2,2)$, this can be easily verified for curves of degree $d \leqq 7, d \leqq 5$ and $d \leqq 4$, resp.

We come back to the restriction problem. Not every stable deformation of $\mathscr{T}_{X} \oplus$ $\mathcal{O}_{X}$ is equally interesting for physicists. They ask for deformations whose restriction to a rational curve is not trivial, i.e. not isomorphic to $\mathcal{O}^{\oplus 4}$. Every vector bundle on $\mathbf{P}_{1}$ can be written as $\oplus \mathcal{O}\left(a_{i}\right)$ and $\left(a_{1}, \ldots, a_{r}\right)$ is called its splitting type. For vector bundles with trivial determinant, $\left(a_{1}, \ldots, a_{r}\right)=(0, \ldots, 0)$ is the generic splitting type. So we are interested in stable deformations of $\mathscr{T}_{X} \oplus \mathcal{O}_{X}$ with non-generic splitting type on each rational curve. First of all, we recall that neither $\mathscr{T}_{X} \oplus \mathcal{O}_{X}$ nor $F$ can have generic splitting type on a rational curve. Let $\varphi: \mathbf{P}_{1} \longrightarrow X$ be a non-constant morphism. Then the injection $\mathscr{T}_{\mathbf{P}_{1}} \hookrightarrow \varphi^{*} \mathscr{T}_{X}$ excludes the triviality of $\varphi^{*} \mathscr{T}_{X}$, and hence of $\varphi^{*}\left(\mathscr{T}_{X} \oplus \mathcal{O}_{X}\right)$, since $\mathscr{T}_{\mathbf{P}_{1}} \cong \mathcal{O}_{\mathbf{P}_{1}}(2)$. The pull-back $\varphi^{*} \omega \in H^{1}\left(\mathbf{P}_{1}, \Omega_{\mathbf{P}_{1}}^{1}\right)$ of the Kähler class defining $F$ can be interpreted as a non-trivial extension class giving rise to the exact sequence

$$
0 \longrightarrow \mathcal{O}_{\mathbf{P}_{1}} \longrightarrow \mathcal{O}_{\mathbf{P}_{1}}(1) \oplus \mathcal{O}_{\mathbf{P}_{1}}(1) \longrightarrow \mathcal{O}_{\mathbf{P}_{1}}(2) \longrightarrow 0 .
$$

Therefore, there is an injection $\mathcal{O}_{\mathbf{P}_{1}}(1) \oplus \mathcal{O}_{\mathbf{P}_{1}}(1) \longrightarrow F$ being part of the commutative diagram

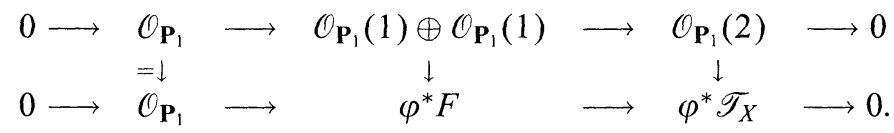


Again, this excludes the triviality of $\varphi^{*} F$. In general, there is no obvious reason why not every deformation of $\mathscr{T}_{X}$ or $F$ should be trivial. In fact one can prove the following.

Proposition 3.2. If $X$ is a smooth quintic hypersurface in $\mathbf{P}_{4}$, then the generic deformation of $\tilde{T}_{X} \oplus \mathcal{O}_{X}$ has trivial restriction to any given rational curve $\varphi$ : $\mathbf{P}_{1} \rightarrow X$.

Proof. Of course, it is enough to produce a deformation of $\mathscr{T}_{X} \oplus \mathcal{O}_{X}$ or $F$, resp. that restricts trivially, i.e. the pull-back under $\varphi$ is isomorphic to the trivial rank four bundle. The idea is to use $\left.\Omega_{\mathbf{P}_{4}}\left\langle X^{\prime}\right\rangle\right|_{X}$ as a deformation of $F^{*}$, where $X^{\prime}$ is the union of the coordinate hyperplanes $H_{0}, H_{1}, \ldots, H_{4}$. As in Sect. 1 we have an exact sequence

$$
0 \longrightarrow \Omega_{\mathbf{P}_{4}} \longrightarrow \Omega_{\mathbf{P}_{4}}\left\langle X^{\prime}\right\rangle \longrightarrow \oplus \mathcal{O}_{H_{l}} \longrightarrow 0
$$

Analogously to the Euler sequence there also exists

$$
0 \longrightarrow \Omega_{\mathbf{P}_{4}}\left\langle X^{\prime}\right\rangle \longrightarrow \oplus^{5} \mathcal{O}_{\mathbf{P}_{4}} \longrightarrow \mathcal{O}_{\mathbf{P}_{4}} \longrightarrow 0
$$

locally given by $d\left(x_{i} / x_{0}\right) /\left(x_{i} / x_{0}\right) \mapsto e_{i}-e_{0}$. Hence $\Omega_{\mathbf{P}_{4}}\left\langle X^{\prime}\right\rangle \cong 4 \mathcal{O}_{\mathbf{P}_{4}}$. In particular, $\varphi^{*} \Omega_{\mathbf{P}_{4}}\left\langle X^{\prime}\right\rangle \cong 4 \mathcal{O}_{\mathbf{P}_{1}}$. Unfortunately, $\left.\Omega_{\mathbf{P}_{4}}\left\langle X^{\prime}\right\rangle\right|_{X}$ does not represent a deformation of $F^{*}$ if $X^{\prime}$ is not smooth. Instead of $\left.\Omega_{\mathbf{P}_{4}}\left\langle X^{\prime}\right\rangle\right|_{X}$ consider $\alpha^{-1}\left(\mathcal{O}_{X^{\prime} \cap X}\right)$, where $\alpha:\left.\Omega_{\mathbf{P}_{4}}\left\langle X^{\prime}\right\rangle\right|_{X} \rightarrow \oplus \mathcal{O}_{H_{l}} \mid X$ and $\mathcal{O}_{X \cap X^{\prime}}$ is considered as a subsheaf of $\oplus \mathcal{O}_{H_{1}}$. The sheaf $\alpha^{-1}\left(\mathcal{O}_{X^{\prime} \cap X}\right)$ is a flat deformation of $F^{*}$, though not locally free, and if the coordinates $x_{i}$ are chosen such that $\varphi\left(\mathbf{P}_{1}\right) \cap H_{i} \cap H_{j}=\emptyset(i \neq j)$, then $\varphi^{*} \alpha^{-1}\left(\mathcal{O}_{X^{\prime} \cap X}\right) \cong$ $\left.\varphi^{*} \Omega_{\mathbf{P}_{4}}\left\langle X^{\prime}\right\rangle\right|_{X} \cong 4 \mathcal{O}_{\mathbf{P}_{1}}$.

More in the vein of Sect. 2 one can also argue as follows. The bundle $F$ lives in fact on $\mathbf{P}_{4}$ and is there isomorphic to the kernel of

$$
\left(\frac{\partial f_{0}}{\partial x_{i}}\right): \oplus^{5} \mathcal{O}(1) \longrightarrow \mathcal{O}(5) \text {. }
$$

This degenerates to $\left(\partial\left(x_{0} x_{1} x_{2} x_{3} x_{4}\right) / \partial x_{i}\right)$. Via $\left(a_{0}, \ldots, a_{3}\right) \mapsto\left(a_{0} x_{0}, \ldots, a_{3} x_{3}\right.$, $\left.\left(-a_{0}-\ldots-a_{3}\right) x_{4}\right)$ its kernel is isomorphic to 40 . As above, it needs a slight modification to obtain a flat deformation of $F$.

Comparing the results of (3.1) and (3.2) in the case of a generic quintic we see that in the commutative diagram

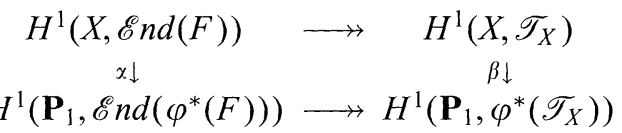

the map $\alpha$, which describes the tangent map of $\operatorname{Def}(F) \rightarrow \operatorname{Def}\left(\varphi^{*}(F)\right)$, is never zero, whereas $\beta$ always vanishes. The surjectivity of $\alpha$ for all rational curves would imply Clemens' conjecture.

Theorem 3.3. $i$ ) For the generic quintic hypersurface $X \subset \mathbf{P}_{4}$ the bundle $\mathscr{T}_{X} \oplus \mathcal{O}_{X}$ can be deformed to a stable bundle whose restriction to every rational curve of degree one, i.e. a line, is nontrivial.

ii) For any quintic $X \subset \mathbf{P}_{4}$ and a fixed curve $\varphi: \mathbf{P}_{1} \rightarrow X$ of degree $d<20$, the bundle $\mathscr{T}_{X} \oplus \mathcal{O}_{X}$ admits a stable deformation, such that the pull-back under $\varphi$ is not trivial. 
Proof. $i$ ) Let $\varphi: \mathbf{P}_{1} \longrightarrow X \subset \mathbf{P}_{4}$ be a non-constant map of degree $d$. By the same arguments we used to prove the nontriviality of $\varphi^{*} F$ we can also derive the following diagram:

$$
\begin{array}{cccccc}
\mathcal{O}_{\mathbf{P}_{1}} \oplus \mathcal{O}_{\mathbf{P}_{1}} & \subset & \varphi^{*}(F)(-1) & \subset & \oplus^{5} \mathcal{O}_{\mathbf{P}_{1}}(d-1) \\
\downarrow & & \downarrow & & & \downarrow \\
\mathscr{T}_{\mathbf{P}_{1}}(-1) & \subset & \varphi^{*}\left(\mathscr{T}_{X}\right)(-1) & \subset & \varphi^{*}\left(\mathscr{T}_{\mathbf{P}_{4}}\right)(-1) .
\end{array}
$$

Thus we obtain two sections $s_{1}, s_{2} \in H^{0}\left(\mathbf{P}_{1}, \oplus^{5} \mathcal{O}_{\mathbf{P}_{1}}(d-1)\right)$. If $d=1$, there is a nontrivial linear combination $a_{1} s_{1}+a_{2} s_{2}\left(a_{i} \in \mathbb{C}\right)$, an element of $\oplus^{5} H^{0}\left(\mathbf{P}_{1}, \mathcal{O}_{\mathbf{P}_{1}}\right)$, whose first component vanishes. Deforming the equation $f$ of $X$ to $f^{\prime}=f+\lambda x_{0}^{5}$ will change the isomorphism type of $X$, if $X$ was chosen generically. In particular, the restriction of the associated bundle $F_{f^{\prime}}$ to $X$ defines a stable deformation of $F$. Since $\varphi^{*}\left(\left(\partial f^{\prime} / \partial x_{i}\right)\right)\left(a_{1} s_{1}+a_{2} s_{2}\right)=\varphi^{*}\left(\left(\partial f / \partial x_{l}\right)\right)\left(a_{1} s_{1}+a_{2} s_{2}\right)+\varphi^{*}\left(5 x_{0}^{4}\right)\left(a_{1} s_{1}+\right.$ $\left.a_{2} s_{2}\right)_{1}=0, a_{1} s_{1}+a_{2} s_{2}$ is in fact a section of $\varphi^{*}\left(F_{f^{\prime}}\right)(-1) \subset \oplus^{5} \mathcal{O}_{\mathbf{P}_{1}}$, too. This contradicts $\varphi^{*}\left(F_{f^{\prime}}\right) \cong \mathcal{O}^{\oplus 4}$.

ii) Let $C$ be the image of $\varphi$ and $I_{C}$ be the ideal sheaf of $C$ in $\mathbf{P}_{4}$. Using the exact sequence

$$
0 \longrightarrow H^{0}\left(\mathbf{P}_{4}, I_{C}(5)\right) \longrightarrow H^{0}\left(\mathbf{P}_{4}, \mathcal{O}(5)\right) \longrightarrow H^{0}\left(C, \mathcal{O}_{C}(5)\right) \longrightarrow
$$

we have $h^{0}\left(\mathbf{P}_{4}, I_{C}(5)\right) \geqq h^{0}\left(\mathbf{P}_{4}, \mathcal{O}(5)\right)-h^{0}\left(C, \mathcal{O}_{C}(5)\right) \geqq h^{0}\left(\mathbf{P}_{4}, \mathcal{O}(5)\right)-h^{0}\left(\mathbf{P}_{1}, \mathcal{O}_{\mathbf{P}_{1}}\right.$ $(5 d))=126-(5 d+1)$. On the other hand, $h^{0}\left(X,\left.\mathscr{T}_{\mathbf{P}_{4}}\right|_{X}\right)=24$ and $24<$ $126-(5 d+1)-1$ by assumption. Hence $H^{0}\left(\mathbf{P}_{4}, I_{C}(5)\right)$ under the restriction map $H^{0}\left(\mathbf{P}_{4}, \mathcal{O}(5)\right) \rightarrow H^{0}\left(X, \mathcal{O}_{X}(5)\right)$ cannot be completely contained in the subspace $H^{0}\left(X, \mathscr{T}_{\mathbf{P}_{4}} \mid X\right)$ which is the kernel of $H^{0}\left(X, \mathcal{O}_{X}(5)\right) \rightarrow H^{1}\left(X, \mathscr{T}_{X}\right)$. Thus we can find a small non-isomorphic deformation $X^{\prime}$ of $X$, such that $C$ is still contained in $X^{\prime}$. As we have seen, $\Omega_{\mathbf{P}_{4}}\left\langle X^{\prime}\right\rangle$ restricts non-trivially to any rational curve contained in $X^{\prime}$. On the other hand $\left.\Omega_{\mathbf{P}_{4}}\left\langle X^{\prime}\right\rangle^{*}\right|_{X}$ is a stable deformation of $F$.

Remark. Yet, there is another way to attack the restriction problem in the case of the quintic. We want to mention it briefly. As shown, all small deformations $F^{\prime}$ of $F$ are given as the kernel of a surjection $\oplus^{5} \mathcal{O}_{X}(1) \rightarrow \mathcal{O}_{X}(5)$. If $\left.F^{\prime *} \cong \Omega_{\mathbf{P}_{4}}\left\langle X^{\prime}\right\rangle\right|_{X}$, where $X^{\prime}$ is a smooth quintic near $X$, this homomorphism was given by $\left(\partial f / \partial x_{i}\right)$. One can use these derivatives to define a morphism $\psi: X \rightarrow \mathbf{P}_{4}$. Then $F^{\prime} \cong \psi^{*}\left(\Omega_{\mathbf{P}_{4}}(1)\right) \otimes \mathcal{O}_{X}(1)$. In particular, the restriction of $F^{\prime}$ to a rational curve $\varphi: \mathbf{P}_{1} \rightarrow X$ of degree $d$ is trivial if and only if $(\psi \circ \varphi)^{*}\left(\Omega_{\mathbf{P}_{4}}(1)\right) \cong 5 \mathcal{O}_{\mathbf{P}_{1}}(-4 d)$. Let the numbers $a_{l}$ be defined by $(\psi \circ \varphi)^{*}\left(\Omega_{\mathbf{P}_{4}}(1)\right) \cong \oplus \mathcal{O}_{\mathbf{P}_{1}}\left(-a_{i}\right)$. Then $\Sigma a_{i}=4 d$ and $0 \leqq a_{1} \leqq \cdots \leqq a_{4}$ ([Ra]). The general philosophy says that $\left(a_{1}, \ldots, a_{4}\right) \neq(d, \ldots, d)$ if $\psi \circ \varphi$ maps $\mathbf{P}_{1}$ to a special hypersurface, e.g. it maps to a hyperplane if and only if $a_{1}=0$. To construct deformations of $F$ with non-trivial restriction to all rational curves it would be convenient to find a deformation $X^{\prime}$ of $X$ such that the associated morphism $\psi$ : $X \rightarrow \mathbf{P}_{4}$ has an image which is special enough.

Example. Let $X$ be the singular quintic $H_{0} \cup \ldots \cup H_{4}=Z\left(x_{0} x_{1} x_{2} x_{3} x_{4}\right)$ and let $X^{\prime}$ be defined by $f^{\prime}=t \cdot x_{0}^{5}+x_{0} x_{1} x_{2} x_{3} x_{4}$. Let $y_{0}, \ldots, y_{4}$ be the coordinates of $\mathbf{P}_{4}$ such that $\psi^{*} y_{i}=\frac{\partial f^{\prime}}{\partial x_{1}}$. Since $X \subset Z\left(\frac{\partial f^{\prime}}{\partial x_{1}} \cdot \frac{\partial f^{\prime}}{\partial x_{2}}\right)$, the image of $\psi$ is contained in the union of the two hyperplanes defined by $y_{1}$ and $y_{2}$. Thus for any rational curve $\varphi: \mathbf{P}_{1} \rightarrow X$ the bundle $(\psi \circ \varphi)^{*}\left(\Omega_{\mathbf{P}_{4}}(1)\right) \otimes \mathcal{O}_{\mathbf{P}_{1}}(d)$ is never trivial. 


\section{A. Deformation Theory of Manifolds and Bundles}

In this section we want to collect some general facts about the deformation of varieties and vector bundles. Some of them we used tacitly throughout the text.

Let $X$ be a compact complex manifold. Its infinitesimal deformations are parametrized by the vector space $H^{1}\left(X, \mathscr{T}_{X}\right)$. The obstructions live in $H^{2}\left(X, \mathscr{T}_{X}\right)$ and the versal deformation space $\operatorname{De} f(X)$, which always exists, is isomorphic to $\kappa_{X}^{-1}(0)$, where $\kappa_{X}^{-1}: H^{1}\left(X, \mathscr{T}_{X}\right) \longrightarrow H^{2}\left(X, \mathscr{T}_{X}\right)$ is the locally defined Kuranishi map (cf. [Ko])

An analogous theory exists for deformations of $X$ as a submanifold of $Y$. The infinitesimal deformations can be identified with the elements of $H^{0}\left(X, \mathscr{N}_{X / Y}\right)$, where $\mathscr{N}_{X / Y}$ is the normal bundle of $X$ in $Y$. Again, there is an obstruction space which can be identified with $H^{1}\left(X, \mathscr{N}_{X / Y}\right)$ and a Kuranishi map $\kappa_{X / Y}: H^{0}\left(X, \mathscr{N}_{X / Y}\right) \longrightarrow$ $H^{1}\left(X, \mathscr{N}_{X / Y}\right)$, such that $\operatorname{Def}(X \subset Y) \cong \kappa_{X / Y}^{-1}(0)$. These two deformation spaces are related as follows. If

$$
\left.0 \longrightarrow \mathscr{T}_{X} \longrightarrow \mathscr{T}_{Y}\right|_{X} \longrightarrow \mathscr{N}_{X / Y} \longrightarrow 0
$$

is the normal bundle sequence of $X$ in $Y$, then the boundary maps induce the commutative diagram (the Kuranishi maps are locally defined)

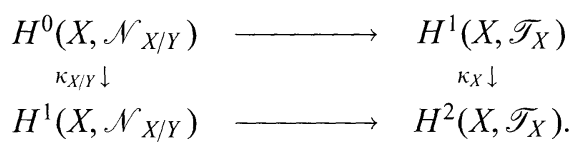

The associated morphism between the kernels $\kappa_{X / Y}^{-1}(0) \rightarrow \kappa_{X}^{-1}(0)$ maps a deformation $X^{\prime} \subset Y$ of $X \subset Y$ to $X^{\prime}$. The Kuranishi description of $\operatorname{De} f(X)$ and $\operatorname{De} f(X \subset Y)$ shows that they are smooth if the obstruction space $H^{2}\left(X, \mathscr{T}_{X}\right)$ resp. $H^{1}\left(X, \mathscr{N}_{X / Y}\right)$ vanishes. We shall say that $X$ (the embedding $X \subset Y$ ) has unobstructed deformations if $\operatorname{Def}(X)$, the versal deformation space of $X$ (Def $(X \subset Y)$ the versal deformation space of the embedding $X \subset Y)$ is smooth. This is equivalent to $\operatorname{dim} \operatorname{Def}(X)=h^{1}\left(X, \mathscr{T}_{X}\right)$ and $\operatorname{dim} \operatorname{Def}(X \subset Y)=h^{0}\left(X, \mathscr{N}_{X / Y}\right)$, resp. In general, we only have $\operatorname{dim} \operatorname{De} f(X) \geqq h^{1}\left(X, \mathscr{T}_{X}\right)-h^{2}\left(X, \mathscr{T}_{X}\right)$ and the corresponding formula for $\operatorname{De} f(X \subset Y)$. In many cases $X$ may have unobstructed deformations, even though the obstruction space does not vanish.

Theorem A.1. $i$ ) If $X$ is a Kähler manifold with $K_{X}^{\otimes n} \cong \mathcal{O}_{X}$ for some $n \neq 0$, then $X$ has unobstructed deformations $[K a, R, T o, T i]$.

ii) Let $X$ be embedded in a compact Kähler manifold $Y$. Then $X \subset Y$ has unobstructed deformations, if the embedding is semiregular [B].

From this one can conclude the following very easily:

Corollary A.2. For every embedding of a Calabi-Yau manifold $X$ in $\mathbf{P}_{N}$ the embedding $X \subset \mathbf{P}_{N}$ has unobstructed deformations.

Proof. One has to use the following trivial fact: If $X$ has unobstructed deformations and $X$ is embedded in $Y$, then $X \subset Y$ has unobstructed deformations if $H^{1}\left(X, \mathscr{N}_{X / Y}\right) \longrightarrow H^{2}\left(X, \mathscr{T}_{X}\right)$ is injective, e.g. if $H^{1}\left(X,\left.\mathscr{T}_{Y}\right|_{X}\right)=0$. This can be 
applied in our situation, since $H^{1}\left(X, \mathscr{T} \mathbf{P}_{N} \mid X\right)=0$, which follows from the Euler sequence and $H^{1}\left(X, \mathcal{O}_{X}(1)\right)=H^{2}\left(X, \mathcal{O}_{X}\right)=0$.

The infinitesimal deformations of a vector bundle $E$ on a fixed compact manifold $X$ are measured by $H^{1}(X, \mathscr{E} n d(E))$ and the obstructions live in $H^{2}(X, \mathscr{E} n d(E))$. The existence of the Kuranishi map $\kappa_{E}: H^{1}(X, \mathscr{E} n d(E)) \longrightarrow H^{2}(X, \mathscr{E} n d(E))$ gives a lower bound for the dimension of the versal deformation space $\operatorname{De} f(E)$ by $h^{1}(X, \mathscr{E} n d(E))-h^{2}(X, \mathscr{E} n d(E))$. In fact, the image of the Kuranishi map is contained in $H^{2}\left(X, \mathscr{E} n d_{0}(E)\right)$, where $\mathscr{E} n d_{0}(E)$ is the sheaf of traceless endomorphisms $[\mathrm{K}]$.

Example: If $E$ is a stable vector bundle on a surface, then $h^{0}(X, \mathscr{E} n d(E))=1$. The Riemann-Roch formula in this case shows $\operatorname{dim} \operatorname{De} f(E) \geqq 4 c_{2}-c_{1}^{2}-4 \chi\left(\mathcal{O}_{X}\right)+1$.

Unfortunately, nothing like this holds for a vector bundle on a Calabi-Yau threefold. Serre duality here gives $h^{1}(X, \mathscr{E} n d(E))=h^{2}(X, \mathscr{E} n d(E))$, i.e. potentially there are as many deformations as obstructions.

We shall say that $E$ has unobstructed deformations, if $\operatorname{De} f(E)$ is smooth. This is equivalent to $\operatorname{dim} \operatorname{De} f(E)=h^{1}(X, \mathscr{E} n d(E))$. The only effective smoothness criterion for $\operatorname{Def}(E)$ is the vanishing of $H^{2}\left(X, \mathscr{E} n d_{0}(E)\right)$. In [R] and [Ka] a more general smoothness criterion is given, but I do not know of any case where it could be checked and $H^{2}\left(X, \mathscr{E} n d_{0}(E)\right)$ does not vanish.

It might also be interesting to consider simultaneous deformations of a variety $X$ and a vector bundle $E$ on $X$. It is not hard to see that in this case the tangent space of the versal deformation space $\operatorname{De} f(X, E)$ is naturally isomorphic to $H^{1}\left(X, \mathscr{D}_{0}^{1}(E)\right)$. The obstructions take values in $H^{2}\left(X, \mathscr{D}_{0}^{1}(E)\right)$. Here $\mathscr{D}_{0}^{1}(E)$ denotes the coherent sheaf of differential operators on $E$ of order $\leqq 1$ whose symbol is contained in the image of the diagonal map $\mathscr{T}_{X} \longrightarrow \mathscr{T}_{X} \otimes \mathscr{E} n \bar{d}(E)$. Using the symbol sequence

$$
0 \longrightarrow \mathscr{E} n d(E) \longrightarrow \mathscr{D}_{0}^{1}(E) \longrightarrow \mathscr{T}_{X} \longrightarrow 0
$$

we get the exact sequence

$$
\begin{aligned}
& \longrightarrow H^{1}(X, \mathscr{E} n d(E)) \longrightarrow H^{1}\left(X, \mathscr{D}_{0}^{1}(E)\right) \longrightarrow H^{1}\left(X, \mathscr{T}_{X}\right) \longrightarrow \\
& \longrightarrow H^{2}(X, \mathscr{E} n d(E)) \longrightarrow H^{2}\left(X, \mathscr{D}_{0}^{1}(E)\right) \longrightarrow H^{2}\left(X, \mathscr{T}_{X}\right) \longrightarrow .
\end{aligned}
$$

The maps between the involved tangent and obstruction spaces naturally commute with the Kuranishi maps. Following an idea of Wilson [Wi] about the deformation of rational curves in the Kuranishi family of $X$ one can prove

Proposition A.3. Let $X$ be a Calabi-Yau threefold and $E$ a vector bundle on $X$ which does not move on $X$, i.e. $\operatorname{dim} \operatorname{De} f(E)=0$, then $E$ deforms with $X$ in the Kuranishi family $X \longrightarrow \operatorname{De} f(X)$, i.e. Def $(X, E) \longrightarrow \operatorname{De} f(X)$, given by mapping a deformation $\left(X^{\prime}, E^{\prime}\right)$ of $(X, E)$ to $X^{\prime}$, is surjective.

Proof. Since a Calabi-Yau manifold has unobstructed deformations, i.e. the Kuranishi map $\kappa_{X}: H^{1}\left(X, \mathscr{T}_{X}\right) \longrightarrow H^{2}\left(X, \mathscr{T}_{X}\right)$ vanishes, the Kuranishi map

$$
\kappa_{(X, E)}: H^{1}\left(X, \mathscr{D}_{0}^{1}(E)\right) \longrightarrow H^{2}\left(X, \mathscr{D}_{0}^{1}(E)\right)
$$


has values in the image of $H^{2}(X, \mathscr{E} n d(E)) \longrightarrow H^{2}\left(X, \mathscr{D}_{0}^{1}(E)\right)$. By the above exact sequence, and using $H^{0}\left(X, \mathscr{T}_{X}\right)=0$ we get

$$
\begin{aligned}
\operatorname{dim} \operatorname{Def}(X, E) \geqq & h^{1}\left(X, \mathscr{D}_{0}^{1}(E)\right)-\left(h^{2}(X, \mathscr{E} n d(E)\right. \\
& \left.-\operatorname{dim} \operatorname{Im}\left(H^{1}\left(X, \mathscr{T}_{X}\right) \longrightarrow H^{2}(X, \mathscr{E} n d(E))\right)\right) \\
= & h^{1}(X, \mathscr{E} n d(E))+h^{1}\left(X, \mathscr{T}_{X}\right)-h^{2}(X, \mathscr{E} n d(E)) \\
= & h^{1}\left(X, \mathscr{T}_{X}\right)=\operatorname{dim} \operatorname{Def}(X) .
\end{aligned}
$$

Since $\operatorname{De} f(X, E) \longrightarrow \operatorname{De} f(X)$ has zero dimensional fibre over the origin, it is in fact surjective.

In [Gr1] Prop.3 Grothendieck proves that, if $E$ is an vector bundle on an arbitrary $X$, such that $H^{2}(X, \mathscr{E} n d(E))=0$, then $E$ deforms with $X$ in the Kuranishi family. For a Calabi-Yau threefold $H^{2}(X, \mathscr{E} n d(E))=0$ is equivalent to $H^{1}(X, \mathscr{E} n d(E))=0$, i.e. $E$ is infinitesimal rigid.

\section{B. Deformation of Sums and Extensions of Stable Vector Bundles}

Let $X$ be a projective manifold with an ample divisor $H$. The slope $\mu(F)$ of a sheaf $F$ of positive rank is defined as the ratio $\left(c_{1}(F) \cdot H^{\operatorname{dim} X-1}\right) / \operatorname{rk}(F)$. A vector bundle $E$ is called polystable if $E$ can be written as a direct sum $E_{1} \oplus \cdots \oplus E_{n}$ of stable vector bundles $E_{l}$ of the same slope. One could ask, under which condition such a polystable bundle deforms to a stable bundle. By the phrase " $E$ deforms to a stable bundle" we mean that there is a connected curve $C$ and a vector bundle $\mathscr{E}$ over $C \times X$ such that for some point $0 \in C$ we have $E \cong \mathscr{E}\{0\} \times X$ and for some other point $t \in C$ the corresponding bundle $\mathscr{E}_{\{t\} \times X}$ is stable. We will restrict here to the case of polystable vector bundles $E$ which are sums of two stable vector bundles. The general deformation theory for such an $E$ provides us with the existence of the semi-universal deformation space $\operatorname{Def}(E)$ and a natural isomorphism between its tangent space and $H^{1}(X, \mathscr{E} n d(E)) \cong H^{1}\left(X, \mathscr{E} n d\left(E_{1}\right)\right) \oplus H^{1}\left(X, \mathscr{E} n d\left(E_{2}\right)\right) \oplus$ $H^{1}\left(X, \mathscr{H}\right.$ om $\left.\left(E_{1}, E_{2}\right)\right) \oplus H^{1}\left(X, \mathscr{H}\right.$ om $\left.\left(E_{2}, E_{1}\right)\right)$.

Proposition B.1. Let $E_{1}$ and $E_{2}$ be two stable vector bundles of the same slope, such that the dimension of $\operatorname{Def}\left(E_{1} \oplus E_{2}\right)$ can be strictly bounded from below by

$$
\operatorname{dim} \operatorname{Def}\left(E_{1}\right)+\operatorname{dim} \operatorname{Def}\left(E_{2}\right)+\max \left\{h^{1}\left(X, \mathscr{H} \operatorname{om}\left(E_{1}, E_{2}\right)\right), h^{1}\left(X, \mathscr{H} \text { om }\left(E_{2}, E_{1}\right)\right)\right\} .
$$

Then $E_{1} \oplus E_{2}$ deforms to a stable bundle.

Proof. We abbreviate $\operatorname{De} f\left(E_{1} \oplus E_{2}\right)$ by $D$. If $\mathscr{E}$ over $X \times D$ is the semi-universal family, such that $\mathscr{E}_{0} \cong E_{1} \oplus E_{2}$, then we can assume that $\mathscr{E}_{t}$ is semistable for all $t \in$ $D$, for semistability is an open property. If $\mathscr{E}_{t}$ is not stable, then there exists a stable subsheaf $0 \neq F \subset \mathscr{E}_{t}$ with $\mu(F)=\mu\left(E_{l}\right)$ and torsionfree cokernel $\mathscr{E}_{t} / F$. For $t=0$ such a subsheaf is automatically isomorphic to one of the summands of $\mathscr{E}_{0} \cong E_{1} \oplus$ $E_{2}$. First, we recall, that there exist finitely many projective schemes $M_{1}, M_{2}, \ldots, M_{n}$ all of which are moduli spaces parametrizing semistable sheaves, such that every stable subsheaf $F$ of $\mathscr{E}_{t}$ corresponds to a point of the union $\cup M_{l}$. Furthermore, we can require, that $E_{1}$ and $E_{2}$ only correspond to points in $M_{1}$ and $M_{2}$, resp. This 
is in fact an application of a result of Grothendieck [Gr2], which says, that the set of Hilbert polynomials of subsheaves $0 \neq F \subset \mathscr{E}_{t}$ with given slope and torsionfree cokernel $\mathscr{E}_{t} / F$ is finite. In particular, there are finitely many projective moduli schemes parametrizing all stable sheaves with these Hilbert polynomials [Ma]. Then we use the incidence varieties $Z_{i}=\left\{\left(F_{l}, t\right) \in M_{i} \times D \mid \operatorname{Hom}\left(F, \mathscr{E}_{t}\right) \neq 0\right\}$, which are closed in $M_{l} \times D$. Since for $i>2$ the image of $Z_{i}$ under the second projection $M_{l} \times D \longrightarrow D$ does not contain 0 , we can assume, by shrinking $D$, that whenever $F$ is a stable subsheaf of $\mathscr{E}_{t}$ with torsionfree cokernel and $\mu(F)=\mu\left(E_{i}\right)$, then $F$ corresponds to a point of the union $M_{1} \cup M_{2}$. To finish the proof we only have to show that under our asumptions the maps $Z_{i=1,2} \longrightarrow D$ are not dominant. We will do it for $i=1$. If $Z_{1} \longrightarrow M_{1}$ is the first projection, then the fibre over $\left[E_{1}\right] \in M_{1}$ is $\left\{t \in D \mid \operatorname{Hom}\left(E_{1}, \mathscr{E}_{t}\right) \neq 0\right\}$. Since any nontrivial homomorphism $E_{1} \rightarrow \mathscr{E}_{t}$ is injective, $\mathscr{E}_{t}$ is in fact given as an extension of the form

$$
0 \longrightarrow E_{1} \longrightarrow \mathscr{E}_{t} \longrightarrow \mathscr{E}_{t} / E_{1} \longrightarrow 0
$$

In addition, for $t=0$ we necessarily have $E_{2} \cong \mathscr{E}_{t} / E_{1}$. Hence, locally around $0 \in D$, the sheaf $\mathscr{E}_{t} / E_{1}$ is a deformation of $E_{2}$. Thus the dimension of the fibre $\left\{t \in D \mid \operatorname{Hom}\left(E_{1}, \mathscr{E}_{t}\right) \neq 0\right\}$ at the point $t=0$ is bounded by

$$
\operatorname{dim} M_{2}+h^{1}\left(X, \mathscr{H} \operatorname{om}\left(E_{2}, E_{1}\right)\right) .
$$

Finally, $\operatorname{dim} Z_{1} \leqq \operatorname{dim} M_{1}+\operatorname{dim} M_{2}+h^{1}\left(X, \mathscr{H} o m\left(E_{2}, E_{1}\right)\right)<\operatorname{dim} D$.

Some immediate consequences are listed in the next corollaries.

Corollary B.2. If $X$ is a K3-surface, then $\mathscr{T}_{X} \oplus \mathcal{O}_{X}$ deforms to a stable bundle.

Corollary B.3. If $E_{1}$ and $E_{2}$ are stable bundles such that their sum has unobstructed deformations and both spaces $H^{1}\left(X, \mathscr{H}\right.$ om $\left.\left(E_{1}, E_{2}\right)\right)$ and $H^{1}(X$, $\mathscr{H}$ om $\left.\left(E_{2}, E_{1}\right)\right)$ do not vanish, then $E_{1} \oplus E_{2}$ deforms to a stable bundle.

Notice, that $E_{1}$ and $E_{2}$ have unobstructed deformations if $E_{1} \oplus E_{2}$ has. The following is well-known.

Corollary B.4. If $X$ is a curve of genus $g>1$, and $E_{1}$ and $E_{2}$ are two stable vector bundles of the same slope, then $E_{1} \oplus E_{2}$ deforms to a stable bundle.

Proof. On a curve any vector bundle has unobstructed deformations. Thus it is enough to check that $H^{1}\left(X, \mathscr{H} \operatorname{om}\left(E_{1}, E_{2}\right)\right) \neq 0$. By Riemann-Roch its dimension is bounded by $\operatorname{rk}\left(E_{1}\right) \cdot \operatorname{rk}\left(E_{2}\right)(g-1)>0$.

Corollary B.5. Let $X$ be a projective variety with $h^{1}\left(X, \mathcal{O}_{X}\right)>4 h^{2}\left(X, \mathcal{O}_{X}\right)$, then $\mathcal{O}_{X} \oplus \mathcal{O}_{X}$ deforms to a stable vector bundle.

Proof. By the assumption $\operatorname{dim} \operatorname{Def}\left(\mathcal{O}_{X} \oplus \mathcal{O}_{X}\right) \geqq 4 h^{1}\left(X, \mathcal{O}_{X}\right)-4 h^{2}\left(X, \mathcal{O}_{X}\right)>3 h^{1}$ $\left(X, \mathcal{O}_{X}\right)=2 \operatorname{dim} \operatorname{Def}\left(\mathcal{O}_{X}\right)+h^{1}\left(X, \mathcal{O}_{X}\right)$.

More generally, the sum $F \oplus F$, where $F$ is a stable vector bundle, deforms to a stable vector bundle if $h^{1}(X, \mathscr{E} n d(F))>4 h^{2}(X, \mathscr{E} n d(F))$. 
Instead of deforming the sum $E_{1} \oplus E_{2}$ directly one could first construct a nontrivial extension

$$
0 \longrightarrow E_{2} \longrightarrow F \longrightarrow E_{1} \longrightarrow 0
$$

given by an extension class $\eta \in \operatorname{Ext}^{1}\left(E_{1}, E_{2}\right) \backslash\{0\}$ and then try to deform the bundle $F$.

Definition B.6. For every deformation of $F$ over a parameter space $D$ we define the map $\xi: T_{D, 0} \longrightarrow \operatorname{Ext}^{1}\left(E_{2}, E_{1}\right)$ as the composition

$$
T_{D, 0} \rightarrow \operatorname{Ext}^{1}(F, F) \rightarrow \operatorname{Ext}^{1}\left(F, E_{1}\right) \rightarrow \operatorname{Ext}^{1}\left(E_{2}, E_{1}\right)
$$

where the last two maps are induced by the above extension.

Proposition B.7. If $E_{1}$ and $E_{2}$ are two stable bundles of the same slope but with $\operatorname{rk}\left(E_{1}\right) \neq \operatorname{rk}\left(E_{2}\right)$ and if $F$ admits a deformation over an one-dimensional parameter space $D$ such that $\xi \neq 0$, then $E_{1} \oplus E_{2}$ deforms to a stable bundle.

Proof. As in the proof of B.1 it is enough to show that all small neighbourhoods $U$ of $0 \in \operatorname{Def}\left(E_{1} \oplus E_{2}\right)$ contain a point $t \in U$, such that the corresponding vector bundle $F_{t}$ does not admit any non-trivial homomorphisms $E_{1}^{\prime} \rightarrow F_{t}$ or $E_{2}^{\prime} \rightarrow F_{t}$ for small deformations $E_{1}^{\prime}$ and $E_{2}^{\prime}$ of $E_{1}$ and $E_{2}$, resp. By the assumption about the rank we can assume that $\operatorname{Hom}\left(E_{1}^{\prime}, F\right)=0$ for all small deformations $E_{1}^{\prime}$ of $E_{1}$. Hence, after shrinking $D$, one may as well assume that $\operatorname{Hom}\left(E_{1}^{\prime}, F^{\prime}\right)=0$ for all deformations $F^{\prime}$ of $F$ parametrized by $D$. Since every open neighbourhood $U$ contains a point $t$ with $F_{t} \cong F$, we can regard such an $F^{\prime}$ also as a deformation of $E_{1} \oplus E_{2}$. If $\operatorname{Hom}\left(E_{2}^{\prime}, F^{\prime}\right)=0$, then $F^{\prime}$ is stable. Otherwise, it can be written as an extension

$$
0 \longrightarrow E_{2}^{\prime} \longrightarrow F^{\prime} \longrightarrow E_{1}^{\prime} \longrightarrow 0
$$

where $E_{1}^{\prime}$ and $E_{2}^{\prime}$ are deformations of $E_{1}$ and $E_{2}$, resp. Note that there is only one (up to scalars) homomorphism $E_{2} \rightarrow F$. Thus it is enough to show that $\xi: T_{P, 0} \longrightarrow$ $\operatorname{Ext}^{1}\left(E_{2}, E_{1}\right)$ vanishes, where $P$ is the space parametrizing all the extensions of the form $(*)$. This can be easily concluded using the exact sequence

$$
\operatorname{Ext}^{1}\left(E_{1}, E_{2}\right) \longrightarrow T_{P, 0} \longrightarrow \operatorname{Ext}^{1}\left(E_{1}, E_{1}\right) \oplus \operatorname{Ext}^{1}\left(E_{2}, E_{2}\right)
$$

and the following commutative diagram:

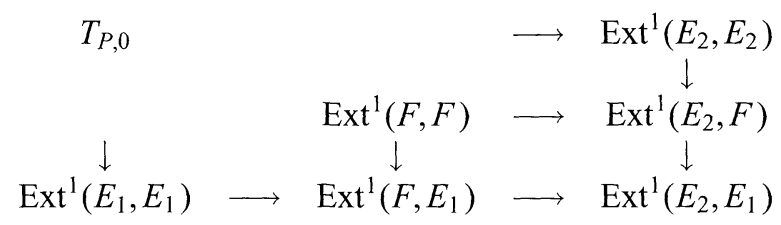

Acknowledgements. It is a pleasure to thank Keiji Oguiso for many enlightening discussions and Manfred Lehn for valuable comments. I am grateful for hospitality and support provided by the Max-Planck-Institut für Mathematik. 


\section{References}

[B] Bloch, S.: Semi-regularity and de Rham cohomology. Inv. Math. 17, 51-66 (1972)

[C] Clemens, H.: Some results about Abel-Jacobi mappings. In: Topics in Transcendental Algebraic Geometry, Annals of Math. Studies, Princeton NJ: Princeton University Press, (1984)

[DGKM] Distler, J., Greene, B. R., Kirklin, K., Miron, P.: Calculating Endomorphism Valued Cohomology: Singlet Spectrum in Superstring Models. Commun. Math. Phys. 122, $117-$ 124 (1989)

[EH] Eastwood, M. G., Hübsch, T.: Endomorphism Valued Cohomology and Gauge-Neutral Matter. Commun. Math. Phys. 132, 383 (1990).

[Grl] Grothendieck, A.: Géométrie formelle et géométrie algébrique. Sém. Bourbaki 182 (1959)

[Gr2] Grothendieck, A.: Techniques de construction et théorem d'existence en géometrie algébrique IV: Les schémas de Hilbert. Sém. Bourbaki 221 (1960/61)

[K1] Katz, S.: On the finiteness of rational curves in quintic threefolds. Comp. Math. 60, 151-162 (1986)

[Ka] Kawamata, Y.: Unobstructed deformations-A remark on a paper of Z. Ran. J. Alg. Geom. 1, 183-190 (1992)

[KJ] Kleiman, S. L., Johnson, T.: On Clemens'conjecture. Preprint (1993)

[K] Kobayashi, S. Differential Geometry of Complex Vector Bundles. Iwanami Shoten, 1987

[Ko] Kodaira, K.: Complex Manifolds and Deformations of Complex Structures. Grundlehren Math. Wiss. 189, Berlin, Heidelberg, New York: Springer 1985

[Ma] Maruyama, M.: Moduli of stable sheaves I. J. Math. Kyoto Univ. 17, 91-126 (1977)

[Mi] Miyaoka, Y.: The Chern Classes and Kodaira Dimension of a Minimal Variety. Adv. Stud. Pure Math. 10, 449-476 (1987)

[N] Nijsse, P.G.J.: Clemens' conjecture for octic and nonic curves. Preprint (1993)

[No] Norten, A.: Analytic moduli of complex vector bundles. Indiana Univ. Math. J. 28, 365-387 (1979)

[R] Ran, Z.: Deformations of manifolds with torsion or negative canonical bundles. J. Alg. Geom. 1, 279-291 (1992)

[Ra] Ramella, L.: La stratification du schéma de Hilbert des courbes rationnelles de $\mathbf{P}^{n}$ par le fibré tangent restreint. C. R. Acad. Sci. Paris 311, I 181-184 (1990)

[Ti] Tian, G.: Smoothness of the universal deformation space of compact Calabi-Yau manifolds and its Petersson-Weil metric. In: Yau, S.- T. Mathematical Aspects of String theory. Singapore: World Scientific, pp. 629-646 1987

[Ti2] Tian, G.: On stability of Fano varieties. Int. J. Math. 3(3), 401-413 1992

[To] Todorov, A. N.: The Weil-Petersson geometry of the moduli space of $S U(n \geqq 3)$ (Calabi-Yau) manifolds. Commun. Math. Phys. 126, 325-346 (1989)

[Ts] Tsuji, H.: Stability of tangent bundles of minimal algebraic varieties. Topology 27, 4 429-442 (1988)

[Wi] Wilson, P. M. H.: The Kähler cone on Calabi-Yau threefolds. Invent. Math. 107, 561$583(1992)$

[W] Witten, E.: New issues in manifolds of $S U(3)$ holonomy. Nucl. Phys. B268, 79-112 (1986)

[Y] Yau, S.-T.: Open Problems in Geometry. Proc. Symp. Pure Math. 54, 1-28 (1993) 